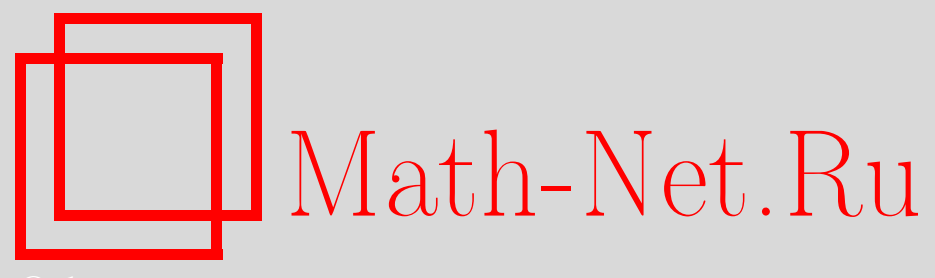

3. С. Ложкина, Об оптимальных точных покрытиях графа в классе слабо плотных базисов, Дискрет. матем., 2004, том 16, выпуск 3, 118-140

DOI: https://doi.org/10.4213/dm167

Использование Общероссийского математического портала Math-Net.Ru подразумевает, что вы прочитали и согласны с пользовательским соглашением http: //www . mathnet.ru/rus/agreement

Параметры загрузки:

IP : 54.237 .206 .68

26 апреля 2023 г., 15:23:48

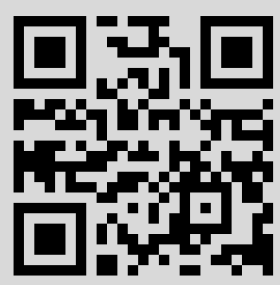




\title{
Об оптимальных точных покрытиях графа в классе слабо плотных базисов
}

\author{
() 2004 г. 3. С. Ложкина
}

\begin{abstract}
Рассматривается задача о покрытии неориентированного связного графа без петель и кратных ребер графами из произвольных конечных базисов. Вводятся понятия сложности покрытия, сложности графа и функции Шеннона. В зависимости от асимптотики функции Шеннона выделены два класса базисов - почти плотные и слабо плотные. Для класса слабо плотных базисов и некоторого специального его подкласса найдены методы построения оптимальных точных покрытий графа двудольными базисными графами и получены оценки сложности таких покрытий. Данные методы основаны на алгоритмах оптимального точного покрытия $(0,1)$-матриц произвольными $(0,1)$-матрицами, а также на соответствии между покрытием $(0,1)$-матрицы и покрытием графа двудольными графами.
\end{abstract}

\section{1. Определения и обозначения}

Пусть $\mathscr{G}^{*}$ - множество всех непустых графов без петель и кратных ребер, $\mathscr{G}$ - множество всех связных графов без петель и кратных ребер, имеющих хотя бы одно ребро, $\mathscr{H}^{*}$ - множество всех двудольных графов без кратных ребер с непустыми долями, а $\mathscr{H}$ множество всех связных двудольных графов без кратных ребер, имеющих хотя бы одно ребро. Очевидно, что $\mathscr{H} \subset \mathscr{G}_{\mathcal{L}} \mathscr{G}^{*}$ и $\mathscr{H} \subset \mathscr{H}^{*}$.

Граф, состоящий из двух вершин и одного ребра, соединяющего эти вершины, назовем тривиальным. Число ребер графа $G$ будем обозначать через $q(G)$, а число его вершин через $p(G)$.

Множество попарно различных графов $B=\left\{G_{1}, \ldots, G_{s}\right\}$ с числами ребер соответственно $r_{1}, \ldots, r_{s}$ называется базисом, если оно содержит тривиальный граф и $G_{i} \in G_{\text {при }}$ любом $i=1,2, \ldots, s$. Весом базиса $B$ называется величина

$$
\rho_{B}=\max _{1 \leqslant i \leqslant s} r_{i}
$$

Очевидно, что для любого базиса $B$ выполнено неравенство $\rho_{B} \geqslant 1$.

Пусть графы $G, H_{1}, \ldots, H_{m}, m \geqslant 1$, принадлежат классу $\mathscr{G}$. Покрытием графа $G$ графами $H_{1}, \ldots, H_{m}$ называется такое взаимно однозначное соответствие между графами $H_{1}, \ldots, H_{m}$ и подграфами $G_{1}, \ldots, G_{m}$ графа $G$, при котором каждое ребро графа $G$ принадлежит по крайней мере одному из подграфов $G_{1}, \ldots, G_{m}$ и для любого $i=1, \ldots, m$ граф $G_{i}$ изоморфен графу $H_{i}$. Если подграфы $G_{1}, \ldots, G_{m}$ попарно не пересекаются по ребрам, то покрытие графа $G$ называется точным. 
Будем говорить, что граф $G \in \mathscr{G}$ покрывается (точно покрывается) графами базиса $B$, если существует такое покрытие (соответственно точное покрытие) графа $G$ графами $H_{1}, \ldots, H_{m}$, что $H_{i} \in B$ для любого $i=1,2, \ldots, m$. Сложностью такого покрытия будем называть число входящих в него графов, то есть число $m$.

Минимальное число графов базиса $B$, достаточное для покрытия всех ребер графа $G \in \mathscr{G}$, называется сложностью графа $G$ и обозначается $l_{B}(G)$. Покрытие графа $G$ графами базиса $B$ называется минимальным, если его сложность равна $l_{B}(G)$.

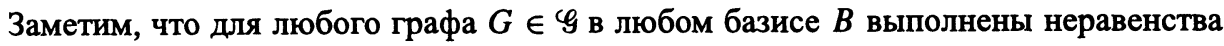

$$
\frac{q(G)}{\rho_{B}} \leqslant l_{B}(G) \leqslant q(G) .
$$

Первое из этих неравенств следует из того, что

$$
q(G) \leqslant \sum_{k=1}^{l_{B}(G)} q\left(H_{k}\right),
$$

где $H_{1}, \ldots, H_{l_{B}(G)}$ - минимальное покрытие графа $G$; второе неравенство вытекает из наличия в базисе $B$ тривиального графа.

Функцией Шеннона в классе $\mathscr{G}$ называется функщия

$$
l_{B}(q, p)=\max _{G \in \mathscr{G}: q(G)=q, p(G)=p} l_{B}(G) .
$$

Из (1.1) и определения функции Шеннона следует, что для любого базиса $B$ и любых натуральных $q, p$ справедливо неравенство

$$
\frac{q}{\rho_{B}} \leqslant l_{B}(q, p) \leqslant q .
$$

Базис $B$ называется почти плотным, если

$$
l_{B}(q, p)=\frac{q}{\rho_{B}}+O(p) .
$$

Базис $B$ называется слабо плотным, если

$$
l_{B}(q, p)=\frac{q}{\rho_{B}}+o\left(p^{2}\right) .
$$

Ранее (см. $[3,4])$ были указаны критерии принадлежности базиса к каждому из перечисленных классов. Так, оказалось, что базис $B$ с весом $\rho$ является почти плотным тогда и только тогда, когда он содержит дерево с $\rho$ ребрами, а слабо плотным - тогда и только тогда, когда он содержит двудольный граф с $\rho$ ребрами. В данной работе описываются методы построения оптимальных точных покрытий графа из класса $\mathscr{G}$ двудольными базисными графами для класса слабо плотных базисов и некоторого специального его подкласса. Эти методы основаны на алгоритмах покрытия $(0,1)$-матриц произвольными $(0,1)$-матрицами. Прежде всего мы введем понятие покрытия $(0,1)$-матрицы, а затем укажем соответствие между покрытием $(0,1)$-матрицы и покрытием графа двудольными графами.

Пусть $G$ - произвольный граф из класса $\mathscr{G}^{*}$, содержащий $p$ вершин $v_{1}, v_{2}, \ldots, v_{p}$. Матрица смежности графа $G$ есть квадратная симметричная матрица $M=M_{p \times p}=\left\|m_{i j}\right\|$, 
в которой $m_{i j}=1$ для $i, j \in[1, p]$, если вершины $v_{i}$ и $v_{j}$ смежны, и $m_{i j}=0$ в противном случае.

Пусть $H=H_{r, t}, r, t \geqslant 1,-$ двудольный граф из класса $\mathscr{H}^{*}$, содержащий $r$ вершин в первой доле и $t$ вершин во второй доле. Сокращенной матрищей смежности графа $H$ называется матрища $A=A_{r \times t}=\left\|a_{i j}\right\|$, в которой $a_{i j}=1$ для $i \in[1, r]$ и $j \in[1, t]$, если $i$-я вершина первой доли смежна с $j$-й вершиной второй доли, и $a_{i j}=0$ в противном случае.

Матрицу, состоящую из нулей и единиц, будем называть $(0,1)$-матрицей. Через $e(M)$ обозначим число единиц, а через $z(M)$ - число нулей в $(0,1)$-матрице $M$. Матрицу, состоящую из одних единиц, будем называть тождественной. Через $E_{r, t}$ обозначим тождественную матрицу, состоящую из $r$ строк и $t$ столбцов. Очевидно, что для любого полного двудольного графа $K_{r, t}, r, t \geqslant 1$, сокращенная матрица смежности является тождественной.

Пусть $M=M_{p \times p}=\left\|m_{i j}\right\|$ - квадратная матрица, $p \geqslant 1$. Верхним сокращенным треугольником матрицы $M$ называется множество элементов $\left\{m_{i j}: j>i\right\}$, а нижним сокращенным треугольником - множество элементов $\left\{m_{i j}: j<i\right\}$.

Две $(0,1)$-матрицы называются изоморфными, если они получаются друг из друга в результате некоторой перестановки строк и столбцов и, возможно, транспонирования. Для изоморфности $(0,1)$-матриц $A$ и $A^{\prime}$ будем использовать обозначение $A \cong A^{\prime}$.

Будем говорить, что $(0,1)$-матрица $B$ является усечением $(0,1)$-матрицы $D$, если матрица $B$ получается из $D$ заменой некоторого количества единиц на нули.

Пусть $D=D_{m \times n}, m, n \geqslant 1,-(0,1)$-матрица. Усеченной подматрицей матрицы $D$, расположенной в строках с номерами $i_{1}, \ldots, i_{r}$ и столбцах с номерами $j_{1}, \ldots, j_{t}$, где $1 \leqslant r \leqslant m, 1 \leqslant t \leqslant n, 1 \leqslant i_{1}, \ldots, i_{r} \leqslant m, 1 \leqslant j_{1}, \ldots, j_{t} \leqslant n$, называется любое усечение подматрицы матрицы $D$, расположенной в строках с номерами $i_{1}, \ldots, i_{r}$ и столбцах с номерами $j_{1}, \ldots, j_{t}$.

Пусть $D, B_{1}, \ldots, B_{N}-(0,1)$-матрицы, $N \geqslant 1$. Покрытием матрицы $D$ (верхнего сокращенного треугольника $D$ ) матрицами $B_{1}, \ldots, B_{N}$ называется такое взаимно однозначное соответствие между матрицами $B_{1}, \ldots, B_{N}$ и усеченными подматрицами $D_{1}, \ldots, D_{N}$ матрицы $D$ (усеченными подматрицами $D_{1}, \ldots, D_{N}$ матрицы $D$, целиком лежащими в ее верхнем сокращенном треугольнике), при котором любая единица матрицы $D$ (верхнего сокращенного треугольника $D$ ) принадлежит хотя бы одной из усеченных подматриц $D_{1}, \ldots, D_{N}$, и $B_{i} \cong D_{i}$ для любого $i=1, \ldots, N$. Матрица $D_{i}$ называется образом матрицы $B_{i}$, а число $N$ называется сложностью покрытия.

Покрытие $(0,1)$-матрицы $D$ (верхнего сокращенного треугольника $D)(0,1)$-матрицами $B_{1}, \ldots, B_{N}$ называется точным, если образы матриц $B_{1}, \ldots, B_{N}$ в матрице $D$ попарно не пересекаются по единицам.

\section{2. О соотношении между покрытием $(0,1)$-матрицы и покрытием графа двудольными графами}

Лемма 1. Связные двудольные графы из класса Н изоморфны тогда и только тогда, когда изоморфны их сокращенные матричы смежности.

Доказательство. Рассмотрим произвольный связный двудольный граф $H \in \mathscr{H}$. Покажем, что его вершины однозначно разбиваются на две доли. Пусть $v_{1}$ и $v_{2}$-две произвольные вершины в графе $H$. Известно, что в любом двудольном графе нет циклов нечетной 
длины. Значит, в графе $H$ не могут одновременно существовать цепи четной и нечетной длины из вершины $v_{1}$ в вершину $v_{2}$. Если в графе $H$ существует цепь четной длины из вершины $v_{1}$ в вершину $v_{2}$, то вершины $v_{1}$ и $v_{2}$ обязаны лежать в одной доле. Если же в графе $H$ существует цепь нечетной длины из вершины $v_{1}$ в вершину $v_{2}$, то вершины $v_{1}$ и $v_{2}$ обязаны лежать в разных долях. Так как $H$ - связный граф, этим определяется разбиение всех вершин графа $H$ на две доли. Таким образом, для связных двудольных графов из класса $\mathscr{H}$ изоморфизм состоит в сопоставлении их долей и последующей перенумерации вершин внутри долей. Для соответствующих сокращенных матриц смежности это означает возможное транспонирование и последующую перестановку строк и столбцов. Но по определению две $(0,1)$-матрицы изоморфны именно тогда, когда одну из другой можно получить с помощью перестановки строк и столбцов и, возможно, транспонирования. Отсюда следует утверждение леммы.

Через $E(G)$ обозначим множество ребер графа $G$. Если $G$ - двудольный граф, то через $V^{1}(G)$ обозначим множество вершин первой доли, а через $V^{2}(G)$ - множество вершин второй доли графа $G$.

Лемма 2. Пусть граф $G \in \mathscr{G}^{*}$ содержит $p$ вериин, $p \geqslant 1$, и имеет матричу смежности $M=M_{p \times p}$, и пусть верхний сокращенный треугольник матрищы $M$ содержсит усеченную подматричу $A=A_{r \times t}, 1 \leqslant r \leqslant p, 1 \leqslant t \leqslant p$. Тогда граф $G$ содержит двудольный подграф из класса $\mathscr{H}^{*}$, имеющий сокращенную матричу смежности $A$.

Доказательство. Пусть усеченная подматрица $A$ матрицы $M$ расположена в строках с номерами $i_{1}, \ldots, i_{r}$ и столбцах с номерами $j_{1}, \ldots, j_{t}$, где $1 \leqslant i_{1}<\ldots<i_{r} \leqslant p$, $1 \leqslant j_{1}<\ldots<j_{t} \leqslant p$. Матрица $A$ лежит в верхнем сокращенном треугольнике матрицы $M$, поэтому $i_{r}<j_{1}$, а следовательно, числа $i_{1}, \ldots, i_{r}, j_{1}, \ldots, j_{t}$ попарно различны. Пусть $A=\left\|a_{k s}\right\|$. Обозначим через $v_{i}, i=1, \ldots, p$, вершину графа $G$, соответствующую $i$-й строке матрицы $M$. Пусть $H$ - такой двудольный граф, что $V^{1}(H)=\left\{v_{i_{1}}, \ldots, v_{i_{r}}\right\}$, $V^{2}(H)=\left\{v_{j_{1}}, \ldots, v_{j_{t}}\right\}$, и

$$
E(H)=\left\{\left(v_{i_{k}}, v_{j_{s}}\right): 1 \leqslant k \leqslant r, 1 \leqslant s \leqslant t, a_{k s}=1\right\} .
$$

Очевидно, что граф $H$ принадлежит классу $\mathscr{H}^{*}$, имеет сокращенную матрищу смежности $A$ и является подграфом графа $G$.

Лемма доказана.

Из лемм 1 и 2 вытекает следующее утверждение.

Лемма 3. Пусть $M$ - матрича смежности графа $G \in \mathscr{G}, A$ - сокращенная матрица смежности двудольного графа $H \in \mathscr{H}$. Если верхний сокращенный треугольник матрицы $M$ содержит усеченную подматричу $A^{\prime}$, изоморфную матриче $A$, то граф $G$ содержит двудольный подграф $H^{\prime} \in \mathscr{H}$, изоморфный графу $H$ и имеючий сокращенную матричу смежности $A^{\prime}$.

Теорема 1. Пусть $M$ - матрича смежности графа $G \in \mathscr{G}, A_{i}$ - сокращенная матрича смежности двудольного графа $H_{i} \in \mathscr{H}, i=1, \ldots, N$. Если существует покрытие (точное покрытие) верхнего сокращенного треугольника матрицы М матричами $A_{1}, \ldots, A_{N}$, то существует и покрытие (точное покрытие) графа $G$ графами $H_{1}, \ldots, H_{N}$. 
Доказательство. Пусть взаимно однозначное отображение $F$ представляет собой покрытие верхнего сокращенного треугольника матрицы $M$ матрицами $A_{1}, \ldots, A_{N}$. Тогда в верхнем сокращенном треугольнике матрицы $M$ существуют такие усеченные подматрицы $A_{1}^{\prime}, \ldots, A_{N}^{\prime}$, что $F\left(A_{i}\right)=A_{i}^{\prime}$, любая единища верхнего сокращенного треугольника матрицы $M$ принадлежит хотя бы одной из усеченных подматриц $A_{1}^{\prime}, \ldots, A_{N}^{\prime}$ и $A_{i}^{\prime} \cong A_{i}$ для любого $i=1, \ldots, N$. Отсюда и из лемм 2,3 вытекает, что в графе $G$ существуют такие двудольные подграфы $H_{1}^{\prime}, \ldots, H_{N}^{\prime} \in \mathscr{H}$, что $H_{i}^{\prime} \cong H_{i}$, граф $H_{i}^{\prime}$ имеет сокращенную матрицу смежности $A_{i}^{\prime}, i=1, \ldots, N$, и любое ребро графа $G$ принадлежит хотя бы одному из подграфов $H_{1}^{\prime}, \ldots, H_{N}^{\prime}$. Следовательно, взаимно однозначное отображение $F^{\prime}: H_{i} \leftrightarrow H_{i}^{\prime}, i=1, \ldots, N$, представляет собой покрытие графа $G$.

Если отображение $F$ есть точное покрытие верхнего сокращенного треугольника матрицы $M$ матрицами $A_{1}, \ldots, A_{N}$, то усеченные подматрицы $A_{1}^{\prime}, \ldots, A_{N}^{\prime}$ матрицы $M$ не пересекаются по единицам, поэтому подграфы $H_{1}^{\prime}, \ldots, H_{N}^{\prime}$ графа $G$ не имеют общих ребер. Следовательно, отображение $F^{\prime}$ представляет собой точное покрытие графа $G$ графами $H_{1}, \ldots, H_{N}$.

Теорема доказана.

\section{3. Точное покрытие тождественной матрицы $(0,1)$-матрицами специального вида}

Пусть $e, l$ - целые числа, $l \geqslant 1$. Через $l \mid e$ обозначим тот факт, что $e$ делится на $l$. Пусть $c, k$ - целые числа, $k \geqslant 0$. Через $\{c\}^{k}$ обозначим последовательность $c, c, \ldots, c$ длины $k$. Через $\{*\}^{k}$ обозначим произвольную последовательность из нулей и единищ длины $k$.

Пусть $a=\left(a_{1}, \ldots, a_{n}\right)$ и $b=\left(b_{1}, \ldots, b_{n}\right)$ - строки длины $n, n \geqslant 1$. Строка $\left(a_{1}+b_{1}, \ldots, a_{n}+b_{n}\right)$ называется суммой строк $a, b$ и обозначается $a+b$.

Пусть $a-(0,1)$-строка длины $n$. Если $a$ содержит хотя бы одну единицу, то через $d(a)$ обозначим позицию последней (то есть самой правой) единицы в строке $a$, в противном случае положим $d(a)=n+1$. Через $b(a)$ обозначим число нулей, лежащих левее первой единицы в строке $a$, а через $e(a)$ - число единиц в $a ;(0,1)$-строка называется сплошной, если все ее единицы идут подряд.

Пусть $a-(0,1)$-строка длины $n, n \geqslant 1$, и пусть $l_{1}, l_{2}$ - целые неотрицательные числа. Добавим к $a$ слева $l_{1}$ нулей и справа $l_{2}$ нулей. Полученная строка длины $n+l_{1}+l_{2}$. называется $\left(l_{1}, l_{2}\right)$-расширением строки $a$ и обозначается $\operatorname{ext}_{l_{1}, l_{2}}(a)$.

Пусть $a_{1}, \ldots, a_{r}, r \geqslant 1,-(0,1)$-строки длины $n \geqslant 1, s, l$ - натуральные числа и $\min (s, r)=1$. Положим

$$
g= \begin{cases}\operatorname{ext}_{0,(r-1) l}\left(a_{1}\right)+\operatorname{ext}_{l,(r-2) l}\left(a_{2}\right)+\ldots+\operatorname{ext}_{(r-1) l, 0}\left(a_{r}\right), & s=1, \\ \operatorname{ext}_{0,(s-1) l}\left(a_{1}\right)+\operatorname{ext}_{l,(s-2) l}\left(a_{1}\right)+\ldots+\operatorname{ext}_{(s-1) l, 0}\left(a_{1}\right), & s>1 .\end{cases}
$$

Строка $g$ длины $n+(s r-1) l$ называется агрегацией строк $a_{1}, \ldots, a_{r}$ с параметрами $s, l$ и обозначается $\operatorname{agr}_{l}^{s}\left(a_{1}, \ldots, a_{r}\right)$.

Рассмотрим пример. Пусть $a_{1}=(1001), a_{2}=(0111)$. Тогда

$$
\begin{aligned}
\operatorname{agr}_{1}^{1}\left(a_{1}, a_{2}\right) & =\operatorname{ext}_{0,1}\left(a_{1}\right)+\operatorname{ext}_{1,0}\left(a_{2}\right)=(10010)+(00111)=(10121), \\
\operatorname{agr}_{2}^{2}\left(a_{1}\right) & =\operatorname{ext}_{0,2}\left(a_{1}\right)+\operatorname{ext}_{2,0}\left(a_{1}\right)=(100100)+(001001)=(101101) .
\end{aligned}
$$

Заметим, что для любого натурального $l$ и любой $(0,1)$-строки $a$ выполнено равенство $\operatorname{agr}_{l}^{1}(a)=a$. 
Группа $(0,1)$-строк $a_{1}, \ldots, a_{r}, r \geqslant 1$, называется агрегируемой с параметрами $s, l$, где $s, l$ - натуральные числа, если $\min (s, r)=1,(s r-1) l<d\left(a_{1}\right)-b\left(a_{1}\right)$ и агрегация $\operatorname{agr}_{l}^{s}\left(a_{1}, \ldots, a_{r}\right)$ представляет собой сплошную $(0,1)$-строку. Группа $(0,1)$-строк $a_{1}, \ldots, a_{r}, r \geqslant 1$, называется агрегируемой, если существуют такие натуральные числа $s, l$, что группа $a_{1}, \ldots, a_{r}$ является агрегируемой с параметрами $s, l$. Если $(0,1)$-строка $a$ представляет собой агрегируемую группу, то она называется агрегируемой. Если для $(0,1)$-матрицы $A$ существует разбиение строк на агрегируемые группы, то $(0,1)$-матрица $A$ называется агрегируемой.

Рассмотрим пример. Строка $a=(0101)$ является агрегируемой с параметрами 2, 1 , так как $\operatorname{agr}_{1}^{2}(a)=(01010)+(00101)=(01111)$. Матрица

$$
\left(\begin{array}{llll}
0 & 1 & 0 & 1 \\
1 & 1 & 0 & 0
\end{array}\right)
$$

является агрегируемой, так как ее первая строка агрегируема с параметрами 2, 1, а вторая - с параметрами $1,1$.

Из определения агрегации $(0,1)$-строк с параметрами $s, l$ вытекает следующая лемма.

Лемма 4. Пусть $a=\left(a_{1}, \ldots, a_{n}\right)-(0,1)$-строка длины $n, n \geqslant 1, s, l$ - натуральные числа, $(s-1) l<d(a)-b(a)$, и пусть $g=\operatorname{agr}_{l}^{s}(a)=\left(g_{1}, \ldots, g_{n+(s-1) l}\right)$. Тогда справедливы следуюшие утверждения.

(1) Если g представляет собой $(0,1)$-строку, то $b(g)=b(a), d(g)=d(a)+(s-1) l$.

(2) Для любого $i=1, \ldots, b(a)$ справедливо равенство $g_{i}=a_{i}$.

(3) Для любого $t=0,1, \ldots, s-1$ и любого $k=1, \ldots, l$

$$
g_{b(a)+t l+k}=a_{b(a)+k}+a_{b(a)+l+k}+\ldots+a_{b(a)+t l+k} .
$$

(4) Для любого $i=b(a)+(s-1) l+1, \ldots, d(a)$

$$
g_{i}=a_{i}+a_{i-l}+\ldots+a_{i-(s-1) l} .
$$

(5) Для любого $t=0,1, \ldots, s-1$ и любого $k=0,1, \ldots, l-1$

$$
g_{d(a)+(s-1) l-t l-k}=a_{d(a)-k}+a_{d(a)-l-k}+\ldots+a_{d(a)-t l-k} .
$$

(6) Для любого $i=d(a)+(s-1) l+1, \ldots, n$ справедливо равенство $g_{i}=a_{i}$.

Лемма 5. Пусть $s, l, n-$ натуральные числа, $a=\left(a_{1}, \ldots, a_{n}\right)-(0,1)$-строка длины $n$.

Если $s=1$, то строка а является агрегируемой с параметрами $s, l$ тогда и только тогда, когда а-сплочная $(0,1)$-строка. когда

Если $s \geqslant 2$, то а является агрегируемой с параметрами $s, l$ тогда и только тогда,

$$
a=\{0\}^{b(a)}\left\{\{1\}^{l}\{0\}^{(s-1) l}\right\}^{q}\{1\}^{l}\{0\}^{n-d(a)},
$$

где

$$
q=\frac{d(a)-b(a)-l}{s l} \geqslant 1 .
$$


Доказательство. Первое утверждение следует из равенства $\operatorname{agr}_{l}^{1}(a)=a$.

Перейдем к доказательству второго утверждения. Докажем необходимость.

Пусть $s \geqslant 2, l \geqslant 1$, строка $a$ является агрегируемой с параметрами $s$ и $l$, и пусть $g=\left(g_{1}, \ldots, g_{n+(s-1) l}\right)=\operatorname{agr}_{l}^{s}(a)-$ агрегация строки $a$, представляющая собой сплошную $(0,1)$-строку.

1. Покажем, что $(d(a)-b(a)-l) /(s l) \geqslant 1$. Из определения агрегируемой строки вытекает, что $(s-1) l<d(a)-b(a)$. Поскольку $g$ - сплошная $(0,1)$-строка, из п. 1 леммы 4 вытекает, что

$$
g_{b(a)+1}=g_{b(a)+2}=\ldots=g_{d(a)+(s-1) l}=1 .
$$

Согласно п. 3 той же леммы, для любого $t=0,1, \ldots, s-1$ и любого $k=1, \ldots, l$ выполняется равенство

$$
g_{b(a)+t l+k}=a_{b(a)+k}+a_{b(a)+l+k}+\ldots+a_{b(a)+t l+k} .
$$

Tak кak

$$
\begin{aligned}
& b(a)+t l+k \leqslant b(a)+s l<d(a)-(s-1) l+s l \leqslant d(a)+(s-1) l, \\
& b(a)+t l+k \geqslant b(a)+1,
\end{aligned}
$$

из (3.3) и (3.4) при $t=0$ вытекает, что $g_{b(a)+k}=a_{b(a)+k}=1$ для любого $k=1, \ldots, l$. Отсюда и из (3.3) и (3.4) при $t \geqslant 1$ следует, что $a_{b(a)+l+k}+\ldots+a_{b(a)+t l+k}=0$ для любого $t=1, \ldots, s-1$ и любого $k=1, \ldots, l$. Таким образом,

$$
a_{b(a)+k}= \begin{cases}1, & k=1, \ldots, l, \\ 0, & k=l+1, \ldots, s l .\end{cases}
$$

Согласно п. 5 леммы 4 для любого $t=0,1, \ldots, s-1$ и любого $k=0,1, \ldots, l-1$ выполняется равенство

$$
g_{d(a)+(s-1) l-t l-k}=a_{d(a)-k}+a_{d(a)-l-k}+\ldots+a_{d(a)-t l-k} .
$$

Так как

$$
\begin{aligned}
& d(a)+(s-1) l-t l-k>b(a)+(s-1) l-l+1 \geqslant b(a)+1, \\
& d(a)+(s-1) l-t l-k \leqslant d(a)+(s-1) l
\end{aligned}
$$

из равенств (3.3) и (3.6) при $t=0$ вытекает, что $g_{d(a)+(s-1) l-k}=a_{d(a)-k}=1$ для любого $k=0,1, \ldots, l-1$. Отсюда и из равенств (3.3) и (3.6) при $t \geqslant 1$ следует, что $a_{d(a)-l-k}+\ldots+a_{d(a)-t l-k}=0$ для любых $t=1, \ldots, s-1, k=0,1, \ldots, l-1$. Таким образом,

$$
a_{d(a)-k}= \begin{cases}1, & k=0, \ldots, l-1, \\ 0, & k=l, \ldots, s l-1 .\end{cases}
$$

Поскольку $(s-1) l<d(a)-b(a)$ и $s \geqslant 2$, то $d(a)-b(a)>l$. Следовательно, строка $a$ не является сплошной, так как в противном случае было бы выполнено неравенство $d(a)-b(a) \leqslant l$. Отсюда и из равенств (3.5), (3.7) вытекает, что $b(a)+s l \leqslant d(a)-l$, то есть $(d(a)-b(a)-l) / s l \geqslant 1$. 
2. Положим $q=[(d(a)-b(a)-l) /(s l)], q \geqslant 1$. Покажем, что для любого целого $c=0,1, \ldots, q$ выполнены равенства

$$
a_{b(a)+c s l+k}=1, \quad k=1, \ldots, l,
$$

а для любого $c=0,1 \ldots, q-1$ выполнены равенства

$$
a_{b(a)+c s l+k}=0, \quad k=l+1, \ldots, s l .
$$

Предположим, что для некоторого $c, 0 \leqslant c \leqslant q-1$, выполнено равенство (3.8). Очевидно, что для любого $k=1, \ldots, l$

$$
\begin{aligned}
b(a)+c s l+k+(s-1) l & \geqslant b(a)+(s-1) l+1, \\
b(a)+c s l+k+(s-1) l & \leqslant b(a)+(\dot{q}-1) s l+l+s l-l=b(a)+q s l \\
& \leqslant b(a)+d(a)-b(a)-l<d(a) .
\end{aligned}
$$

Поэтому из п. 4 леммы 4 вытекает, что для любого $k=1, \ldots, l$

$$
g_{b(a)+c s l+(s-1) l+k}=a_{b(a)+c s l+(s-1) l+k}+\ldots+a_{b(a)+c s l+l+k}+a_{b(a)+c s l+k} .
$$

Из равенств (3.8) и (3.3) следует, что последнее слагаемое в правой части (3.10) равно единице и левая часть также равна единице. Значит, $a_{b(a)+c s l+t l+k}=0$ для любого $k=1, \ldots, l$ и любого $t=1, \ldots, s-1$, а следовательно, для данного $c$ выполняется равенство (3.9).

Предположим теперь, что для некоторого $c, 0 \leqslant c \leqslant q-1$, выполнено равенство (3.9). Очевидно, что для любого $k=1, \ldots, l$

$$
\begin{aligned}
& b(a)+(c+1) s l+k>b(a)+(s-1) l+1, \\
& b(a)+(c+1) s l+k \leqslant b(a)+q s l+l \leqslant b(a)+d(a)-b(a)-l+l=d(a) .
\end{aligned}
$$

Поэтому из п. 4 леммы 4 вытекает, что для любого $k=1, \ldots, l$

$$
g_{b(a)+c s l+s l+k}=a_{b(a)+c s l+s l+k}+a_{b(a)+c s l+(s-1) l+k}+\ldots+a_{b(a)+c s l+l+k} .
$$

Из равенств (3.9) и (3.3) следует, что все слагаемые в правой части (3.11), кроме самого первого, равны нулю, а левая часть равна единице. Значит, $a_{b(a)+(c+1) s l+k}=1$ для любого $k=1, \ldots, l$, то есть равенство (3.8) выполняется для $c+1$.

Таким образом, мы показали, что если для некоторого $c, 0 \leqslant c \leqslant q-1$, выполнено равенство (3.8), то для того же самого $c$ выполнено и равенство (3.9), а если для $c$ выполнено равенство (3.9), то для $c+1$ выполнено равенство (3.8). Отсюда, учитывая то, что при $c=0$ равенства (3.8) и (3.9) вытекают из (3.5), получаем, что равенство (3.9) выполняется для любого $c=0,1, \ldots, q-1$, а равенство (3.8) - для любого $c=0,1, \ldots, q$.

3. Из п. 2 следует, что строка $а$ имеет вид

$$
a=\{0\}^{b(a)}\left\{\{1\}^{l}\{0\}^{(s-1) l}\right\}^{q}\{1\}^{l}\{*\}^{r}\{0\}^{n-d(a)},
$$

где $q=[(d(a)-b(a)-l) /(s l)] \geqslant 1, r=d(a)-b(a)-s l q-l$. Используя рассуждения, аналогичные рассуждениям п. 2, и заменяя в них равенство (3.5) на (3.7), мы придем к выводу, что для любого целого $c=0,1, \ldots, q$ выполнено равенство

$$
a_{d(a)-c s l-k}=1, \quad k=0,1, \ldots, l-1,
$$


для любого $c=0,1, \ldots, q-1$ выполнено равенство

$$
a_{d(a)-c s l-k}=0, \quad k=l, \ldots, s l-1,
$$

и строка $a$ имеет вид

$$
a=\{0\}^{b(a)}\{*\}^{r}\{1\}^{l}\left\{\{0\}^{(s-1) l}\{1\}^{l}\right\}^{q}\{0\}^{n-d(a)} .
$$

Из равенств (3.12) и (3.13) вытекает, что выполнено одно из двух условий: либо $r=0$, либо $r \geqslant s l$. Но из определения величины $q$ следует, что

$$
\begin{aligned}
& q>(d(a)-b(a)-l) /(s l)-1, \\
& r<d(a)-b(a)-l-d(a)+b(a)+l+s l=s l,
\end{aligned}
$$

а значит, $r=0$ и $q=(d(a)-b(a)-l) /(s l)$. Отсюда и из равенства (3.12) следует утверждение леммы.

Докажем достаточностъ.

Пусть $s \geqslant 2, l \geqslant 1, q=(d(a)-b(a)-l) /(s l) \geqslant 1$ и строка $a$ имеет вид

$$
a=\{0\}^{b(a)}\left\{\{1\}^{l}\{0\}^{(s-1) l}\right\}^{q}\{1\}^{l}\{0\}^{n-d(a)} .
$$

Очевидно, что $d(a)-b(a) \geqslant s l+l>(s-1) l$. Теперь покажем, что агрегация $g=\operatorname{agr}_{l}^{s}(a)$ представляет собой сплошную $(0,1)$-строку. Из (3.14) вытекают следующие равенства:

$$
\begin{aligned}
& a_{i}=0, \quad i=1, \ldots, b(a), \\
& a_{b(a)+c s l+k}=1, \quad k=1, \ldots, l, \quad c=0,1, \ldots, q \text {, } \\
& a_{b(a)+c s l+k}=0, \quad k=l+1, \ldots, s l, \quad c=0,1, \ldots, q-1, \\
& a_{i}=0, \quad i=d(a)+1, \ldots, n \text {. }
\end{aligned}
$$

Из п. 2 леммы 4 и равенства (3.15) вытекает, что $g_{i}=0$ для любого $i=1, \ldots, b(a)$. Из п. 3 леммы 4 и равенств (3.16), (3.17) при $c=0$ следует, что для любого $t=0,1, \ldots, s-1$ и любого $k=1, \ldots, l$

$$
g_{b(a)+t l+k}=a_{b(a)+k}=1,
$$

а значит, $g_{i}=1$ для любого $i=b(a)+1, \ldots, b(a)+s l$.

Пусть теперь $b(a)+s l+1 \leqslant i \leqslant d(a)$. Представим число $i$ в виде $i=b(a)+c s l+k$, где либо $1 \leqslant c \leqslant q-1$ и $1 \leqslant k \leqslant s l$, либо $c=q$ и $1 \leqslant k \leqslant l$. Тогда из п. 4 леммы 4 и равенств (3.16), (3.17) можно заключить, что

$$
g_{i}=a_{b(a)+c s l+k}+a_{b(a)+c s l+k-l}+\ldots+a_{b(a)+c s l+k-(s-1) l}=1 .
$$

Из п. 5 леммы 4, равенства (3.16) для $c=q$ и равенства (3.17) для $c=q-1$ вытекает, что для любого $t=0,1, \ldots, s-1$ и любого $k=0,1, \ldots, l-1$

$$
g_{d(a)+(s-1) l-t l-k}=a_{d(a)-k}=1,
$$

а значит, $g_{i}=1$ при любом $i=d(a)+1, \ldots, d(a)+(s-1) l$. Из п. 6 леммы 4 и равенства (3.18) следует, что $g_{i}=0$ для любого $i=d(a)+(s-1) l+1, \ldots, n$.

Таким образом, мы показали, что $g=\{0\}^{b(a)}\{1\}^{s l q+l}\{0\}^{n-d(a)}$, то есть строка $g$ является сплошной $(0,1)$-строкой.

Лемма доказана. 
Следствие 1. Если $(0,1)$-строка а является агрегируемой с параметрами $s, l u s \geqslant 2$, то е(а) делится на $l$.

Теорема 2. Пусть $A=A_{r \times t}$ - агрегируемая $(0,1)$-матрича без нулевых строк, содержащая $\rho$ единич. Тогда существует натуральное число $N \leqslant t^{2 r+1}$ такое, что с помощью $N$ матрич А можно точно покрыть тождественную матричу $E_{\rho \times N}$.

Доказательство. Разобьем строки матрицы $A$ на агрегируемые группы $R^{1}, \ldots, R^{m}$, $m \leqslant r$. Пусть группа $R^{i}$ состоит из строк $a_{1}^{i}, \ldots, a_{r^{i}}^{i}$, где $1 \leqslant r^{i} \leqslant r$ и $r^{1}+\ldots+r^{m}=r$, и пусть агрегация $g^{i}=\operatorname{agr}_{l^{i}}^{i}\left(a_{1}^{i}, \ldots, a_{r^{i}}^{i}\right)$ является сплошной $(0,1)$-строкой, причем $\min \left(s^{i}, r^{i}\right)=1$ и $\left(s^{i} r^{i}-1\right) l^{i}<d\left(a_{1}^{i}\right)-b\left(a_{1}^{i}\right), i=1, \ldots, m$. Положим

$$
e^{i}=e\left(g^{i}\right)=s^{i}\left(e\left(a_{1}^{i}\right)+\ldots+e\left(a_{r^{i}}^{i}\right)\right), \quad i=1, \ldots, m .
$$

Очевидно, что $e^{1} / s^{1}+\ldots+e^{m} / s^{m}=e(A)=\rho$ и если $s^{i} \geqslant 2$, то по следствию 1 из леммы $5 l^{i} \mid\left(e^{i} / s^{i}\right)$. Очевидно также, что $e^{i} \geqslant 1$, так как матрица $A$ не содержит нулевых строк. Пусть $N$ - минимальное общее кратное чисел $e^{1}, \ldots, e^{m}$, не меньшее $t$, то есть $N=\operatorname{lcm}\left(e^{1}, \ldots, e^{m}\right)\left\lceil t / \operatorname{lcm}\left(e^{1}, \ldots, e^{m}\right)\right\rceil$, где $\operatorname{lcm}\left(a_{1}, \ldots, a_{n}\right)$ означает наименьшее общее кратное чисел $a_{1}, \ldots, a_{n}$.

Покажем, что с помощью $N$ матриц $A$ можно точно покрыть тождественную матрицу $E_{\rho \times N}$. Через $B^{k}=\left\|b_{x y}^{k}\right\|, k=1, \ldots, N$, обозначим $k$-ю матрицу $A$, участвующую в покрытии. Через $D=\left\|d_{i j}\right\|$ обозначим тождественную матрицу $E_{\rho \times N}$. Построим такое взаимно однозначное соответствие между матрицами $B^{k}$ и усеченными подматрицами $D^{k}$ матрицы $D, k=1, \ldots, N$, при котором любая единица матрицы $D$ принадлежит ровно одной из усеченных подматриц $D^{1}, \ldots, D^{N}$ и $B^{k} \cong D^{k}$ для любого $k=1, \ldots, N$.

Пусть строка с номером $x$ матрицы $A$ принадлежит группе $R^{i(x)}$ и имеет в ней номер $j(x), x=1, \ldots, r, 1 \leqslant i(x) \leqslant m, 1 \leqslant j(x) \leqslant r^{i(x)}$. Для каждой тройки $(k, x, y)$, где $k=1, \ldots, N, x=1, \ldots, r, y=1, \ldots, t$, определим величины $w_{k}(x), u_{k}(x), v_{k}(y)$ следующим образом:

$$
w_{k}(x)= \begin{cases}A, & s^{i(x)}=1 \\ B, & s^{i(x)} \geqslant 2\end{cases}
$$

где

$$
\begin{aligned}
A & =\left(k-(j(x)-1) l^{i(x)}-1\right) \bmod e^{i(x)} \\
B & =l^{i(x)}\left(\left[\frac{k-1}{s^{i(x)} l^{i(x)}}\right] \bmod \left(\frac{e^{i(x)}}{s^{i(x)} l^{i(x)}}\right)\right)+(k-1) \bmod \left(l^{i(x)}\right), \\
u_{k}(x) & =\sum_{q=1}^{i(x)-1} \frac{e^{q}}{s^{q}}+w_{k}(x)+1 \\
v_{k}(y) & =(k+y-2) \bmod N+1
\end{aligned}
$$

Пусть отображение $F: B^{k} \rightarrow D^{k}=\left\|d_{x y}^{k}\right\|$ переводит матрицу $B^{k}$ в такую усеченную подматрицу $D^{k}$ размера $r \times t$ матрицы $D$, расположенную в строках с номерами $u_{k}(1), \ldots, u_{k}(r)$ и столбцах с номерами $v_{k}(1), \ldots, v_{k}(t)$, что $b_{x y}^{k}=d_{x y}^{k}$.

1. Покажем, что такое определение отображения матриц корректно, то есть $1 \leqslant u_{k}(x) \leqslant \rho, 1 \leqslant v_{k}(y) \leqslant N$, при этом $u_{k}\left(x_{1}\right) \neq u_{k}\left(x_{2}\right)$, если $x_{1} \neq x_{2}$, и $v_{k}\left(y_{1}\right) \neq v_{k}\left(y_{2}\right)$, если $y_{1} \neq y_{2}$. 
Пусть $1 \leqslant k \leqslant N, 1 \leqslant x \leqslant r, 1 \leqslant y \leqslant t$. Неравенство $1 \leqslant v_{k}(y) \leqslant N$, очевидно, вытекает из (3.21). Покажем справедливость неравенства $1 \leqslant u_{k}(x) \leqslant \rho$. Если $s^{i(x)}=1$, то из (3.19) следует, что $0 \leqslant w_{k}(x)<e^{i(x)}$. Если же $s^{i(x)} \geqslant 2$, то из (3.19) следует, что

$$
0 \leqslant w_{k}(x)<l^{i(x)}\left(\frac{e^{i(x)}}{s^{i(x)} l^{i(x)}}-1\right)+l^{i(x)}=\frac{e^{i(x)}}{s^{i(x)}} .
$$

Отсюда вытекает, что при любом значения $s^{i(x)}$

$$
0 \leqslant w_{k}(x)<\frac{e^{i(x)}}{s^{i(x)}}
$$

а следовательно,

$$
1 \leqslant u_{k}(x) \leqslant \sum_{q=1}^{i(x)-1} \frac{e^{q}}{s^{q}}+\frac{e^{i(x)}}{s^{i(x)}} \leqslant \sum_{q=1}^{m} \frac{e^{q}}{s^{q}}=\rho .
$$

Таким образом, для любых $k=1, \ldots, N, x=1, \ldots, r, y=1, \ldots, t$ выполнены неравенства

$$
1 \leqslant u_{k}(x) \leqslant \rho, \quad 1 \leqslant v_{k}(y) \leqslant N .
$$

Пусть $1 \leqslant x_{1}, x_{2} \leqslant r$ и $x_{1} \neq x_{2}$. Покажем, что тогда $u_{k}\left(x_{1}\right) \neq u_{k}\left(x_{2}\right)$. Положим $i\left(x_{1}\right)=i_{1}, i\left(x_{2}\right)=i_{2}, j\left(x_{1}\right)=j_{1}, j\left(x_{2}\right)=j_{2}$ и рассмотрим два случая.

В первом случае $i_{1} \neq i_{2}$, то есть строки матрицы $A$ с номерами $x_{1}, x_{2}$ принадлежат разным группам $R^{i_{1}}, R^{i_{2}}$. Без ограничения общности будем считать, что $i_{1}<i_{2}$. Из неравенства (3.22) следует, что $w_{k}\left(x_{2}\right) \geqslant 0$ и $w_{k}\left(x_{1}\right)<e^{i_{1}} / s^{i_{1}}$. Отсюда и из (3.20) вытекает, что

$$
u_{k}\left(x_{2}\right)-u_{k}\left(x_{1}\right)=\sum_{q=i_{1}}^{i_{2}-1} \frac{e^{q}}{s^{q}}+w_{k}\left(x_{2}\right)-w_{k}\left(x_{1}\right) \geqslant \frac{e^{i_{1}}}{s^{i_{1}}}-w_{k}\left(x_{1}\right)>0,
$$

то есть $u_{k}\left(x_{1}\right) \neq u_{k}\left(x_{2}\right)$.

Во втором случае $i_{1}=i_{2}=i$, то есть строки матрицы $A$ с номерами $x_{1}, x_{2}$ принадлежат одной группе $R^{i}$. Следовательно, $j_{1} \neq j_{2}, r^{i} \geqslant 2$ и $s^{i}=1$. Без ограничения общности будем считать, что $j_{1}<j_{2}$. Положим $l^{i}=l, e^{i}=e, b\left(a_{1}^{i}\right)=b, d\left(a_{1}^{i}\right)=d$. Тогда $\left(r^{i}-1\right) l<d-b$.

Предположим, что $u_{k}\left(x_{1}\right)=u_{k}\left(x_{2}\right)$. Тогда из (3.20) вытекает равенство $w_{k}\left(x_{1}\right)=w_{k}\left(x_{2}\right)$. Поскольку $s^{i}=1$, отсюда и из (3.19) следует, что

$$
k-\left(j_{1}-1\right) l \equiv k-\left(j_{2}-1\right) l \bmod e,
$$

а значит, $e \mid\left(j_{2}-j_{1}\right) l$. С другой стороны, $0<\left(j_{2}-j_{1}\right) l \leqslant\left(r^{i}-1\right) l<d-b<e$. Таким образом, предположив, что $u_{k}\left(x_{1}\right)=u_{k}\left(x_{2}\right)$, мы пришли к противоречию.

Пусть теперь $1 \leqslant y_{1}, y_{2} \leqslant t$ и $y_{1} \neq y_{2}$. Покажем, что в этом случае $v_{k}\left(y_{1}\right) \neq v_{k}\left(y_{2}\right)$. Без ограничения общности будем считать, что $y_{1}<y_{2}$. Пусть $v_{k}\left(y_{1}\right)=v_{k}\left(y_{2}\right)$. Тогда из (3.21) вытекает, что $k+y_{1} \equiv k+y_{2} \bmod N$, а значит, $N \mid\left(y_{2}-y_{1}\right)$. С другой стороны, $0<y_{2}-y_{1}<t \leqslant N$. Таким образом, мы пришли к противоречию, а следовательно, $v_{k}\left(y_{1}\right) \neq v_{k}\left(y_{2}\right)$.

2. Покажем, что при отображении $F$ любая единица матрицы $D$ принадлежит ровно одной из усеченных подматриц $D^{1}, \ldots, D^{N}$.

Пусть для некоторых $k_{1}, k_{2}, x_{1}, x_{2}, y_{1}, y_{2}$ таких, что $1 \leqslant k_{1}, k_{2} \leqslant N, 1 \leqslant x_{1}, x_{2} \leqslant r$, $1 \leqslant y_{1}, y_{2} \leqslant t$ и $\left(k_{1}, x_{1}, y_{1}\right) \neq\left(k_{2}, x_{2}, y_{2}\right)$, верно равенство $b_{x_{1}, y_{1}}^{k_{1}}=b_{x_{2}, y_{2}}^{k_{2}}=1$. Докажем, 
что тогда $\left(u_{k_{1}}\left(x_{1}\right), v_{k_{1}}\left(y_{1}\right)\right) \neq\left(u_{k_{2}}\left(x_{2}\right), v_{k_{2}}\left(y_{2}\right)\right)$. Положим $i\left(x_{1}\right)=i_{1}, i\left(x_{2}\right)=i_{2}, j\left(x_{1}\right)=j_{1}$, $j\left(x_{2}\right)=j_{2}$ и рассмотрим два случая.

В первом случае $i_{1} \neq i_{2}$. Без ограничения общности будем считать, что $i_{1}<i_{2}$. Из неравенства (3.22) следует, что $w_{k_{2}}\left(x_{2}\right) \geqslant 0$ и $w_{k_{1}}\left(x_{1}\right)<e^{i_{1}} / s^{i_{1}}$. Отсюда и из (3.20) вытекает, что

$$
u_{k_{2}}\left(x_{2}\right)-u_{k_{1}}\left(x_{1}\right)=\sum_{q=i_{1}}^{i_{2}-1} \frac{e^{q}}{s^{q}}+w_{k_{2}}\left(x_{2}\right)-w_{k_{1}}\left(x_{1}\right) \geqslant \frac{e^{i_{1}}}{s^{i_{1}}}-w_{k_{1}}\left(x_{1}\right)>0,
$$

то есть $u_{k_{1}}\left(x_{1}\right) \neq u_{k_{2}}\left(x_{2}\right)$.

Во втором случае $i_{1}=i_{2}=i$. Без ограничения общности будем считать, что $j_{1} \leqslant j_{2}$. Положим $l^{i}=l, e^{i}=e, s^{i}=s, b\left(a_{1}^{i}\right)=b, d\left(a_{1}^{i}\right)=d, g^{i}=g$. Тогда $\left(s r^{i}-1\right) l<d-b$. Предположим, что $\left(u_{k_{1}}\left(x_{1}\right), v_{k_{1}}\left(y_{1}\right)\right)=\left(u_{k_{2}}\left(x_{2}\right), v_{k_{2}}\left(y_{2}\right)\right)$. Тогда из равенств (3.20), (3.21) следует, что

$$
\begin{aligned}
& w_{k_{1}}\left(x_{1}\right)=w_{k_{2}}\left(x_{2}\right), \\
& k_{1}+y_{1} \equiv k_{2}+y_{2} \quad \bmod N .
\end{aligned}
$$

Рассмотрим два случая. В первом случае $s=1$. Из (3.24) и (3.19) вытекает, что $k_{1}-\left(j_{1}-1\right) l \equiv k_{2}-\left(j_{2}-1\right) l \bmod e$, а следовательно,

$$
e \mid\left(k_{1}-k_{2}+\left(j_{2}-j_{1}\right) l\right) \text {. }
$$

Поскольку $e \mid N$, из (3.25) вытекает, что

$$
e \mid\left(k_{1}-k_{2}+y_{1}-y_{2}\right) \text {. }
$$

Если $x_{1}=x_{2}=x$, то $j_{1}=j_{2}=j, b_{x, y_{1}}^{k_{1}}=b_{x, y_{2}}^{k_{2}}=1$, а значит, $\left|y_{2}-y_{1}\right|<e\left(a_{j}^{i}\right) \leqslant e$. С другой стороны, из (3.26) и (3.27) следует, что $e \mid\left(y_{1}-y_{2}\right)$, значит, $y_{2}=y_{1}$. Поскольку $1 \leqslant k_{1}, k_{2} \leqslant N$, в этом случае из (3.25) вытекает, что $k_{1}=k_{2}$, но это невозможно, так как по нашему предположению $\left(k_{1}, x_{1}, y_{1}\right) \neq\left(k_{2}, x_{2}, y_{2}\right)$. Значит, $x_{1} \neq x_{2}$ и $j_{1}<j_{2}$.

Из (3.26) и (3.27) вытекает, что $y_{2}-y_{1}+\left(j_{2}-j_{1}\right) l=c e$, где $c$ - целое число. Как уже было отмечено выше при введении обозначений, агрегация $g$ строк $a_{1}^{i}, \ldots, a_{r i}^{i}$ представляет собой сплошную $(0,1)$-строку. Пусть $g=\left(g_{1}, \ldots, g_{t+\left(r^{i}-1\right) l}\right)$. Из определения агрегации строк вытекает, что

$$
g \geqslant \operatorname{ext}_{\left(j_{1}-1\right) l,\left(r-j_{1}\right) l}\left(a_{j_{1}}^{i}\right), \quad g \geqslant \operatorname{ext}_{\left(j_{2}-1\right) l,\left(r-j_{2}\right) l}\left(a_{j_{2}}^{i}\right),
$$

а следовательно,

$$
\begin{aligned}
& g_{y_{1}+\left(j_{1}-1\right) l} \geqslant b_{x_{1}, y_{1}+\left(j_{1}-1\right) l-\left(j_{1}-1\right) l}^{k_{1}}=b_{x_{1}, y_{1}}^{k_{1}}, \\
& g_{y_{2}+\left(j_{2}-1\right) l} \geqslant b_{x_{2}, y_{2}+\left(j_{2}-1\right) l-\left(j_{2}-1\right) l}^{k_{2}}=b_{x_{2}, y_{2}}^{k_{2}} .
\end{aligned}
$$

Отсюда, $g_{y_{1}+\left(j_{1}-1\right) l}=g_{y_{2}+\left(j_{2}-1\right) l}=1$ и $\left|y_{2}+\left(j_{2}-1\right) l-y_{1}-\left(j_{1}-1\right) l\right|<e(g)=e$, то есть $\left|y_{2}-y_{1}+\left(j_{2}-j_{1}\right) l\right|<e$. Значит, $c=0$, и $y_{2}=y_{1}-\left(j_{2}-j_{1}\right) l$. Из определения агрегации строк вытекает, что $g \geqslant \operatorname{ext}_{\left(j_{1}-1\right) l,\left(r-j_{1}\right) l}\left(a_{j_{1}}^{i}\right)+\operatorname{ext}_{\left(j_{2}-1\right) l,\left(r-j_{2}\right) l}\left(a_{j_{2}}^{i}\right)$, а следовательно,

$$
g_{y_{1}+\left(j_{1}-1\right) l} \geqslant b_{x_{1}, y_{1}+\left(j_{1}-1\right) l-\left(j_{1}-1\right) l}^{k_{1}}+b_{x_{2}, y_{1}+\left(j_{1}-1\right) l-\left(j_{2}-1\right) l}^{k_{2}}=b_{x_{1}, y_{1}}^{k_{1}}+b_{x_{2}, y_{2}}^{k_{2}}=2 .
$$

5 Дискретная математика, т.16 №3 
Но тогда получается, что агрегация $g$ не является $(0,1)$-строкой, а это противоречит нашему предположению.

Во втором случае $s \geqslant 2$. Тогда $r^{i}=1, j_{1}=j_{2}=1, x_{1}=x_{2}=x$. Из (3.24) и (3.19) следует, что

$l\left(\left\lfloor\frac{k_{1}-1}{s l}\right\rfloor \bmod \frac{e}{s l}-\left\lfloor\frac{k_{2}-1}{s l}\right\rfloor \bmod \frac{e}{s l}\right)=\left(k_{2}-1\right) \bmod l-\left(k_{1}-1\right) \bmod l$.

Если $y_{1}=y_{2}$, то из (3.25) следует, что $k_{1}=k_{2}$, поскольку $1 \leqslant k_{1}, k_{2} \leqslant N$. Отсюда вытекает, что $\left(k_{1}, x_{1}, y_{1}\right)=\left(k_{2}, x_{2}, y_{2}\right)$, но это противоречит нашему предположению. Значит, $y_{1} \neq y_{2}$. Очевидно, что $\left|\left(k_{2}-1\right) \bmod l-\left(k_{1}-1\right) \bmod l\right|<l$, поэтому обе части равенства (3.28) равны нулю. Отсюда следует, что

$$
\begin{gathered}
l \mid\left(k_{2}-k_{1}\right), \\
e /(s l) \mid\left\lfloor\left(k_{2}-1\right) /(s l)\right\rfloor-\left\lfloor\left(k_{1}-1\right) /(s l)\right\rfloor .
\end{gathered}
$$

Так как $l \mid(e / s)$ и $e \mid N$, то $s l \mid N$. Поэтому из (3.25) и (3.29) следует, что $l \mid\left(y_{2}-y_{1}\right)$. Поскольку $b_{x, y_{1}}^{k_{1}}=b_{x, y_{2}}^{k_{2}}=1$ и $y_{2} \neq y_{1}$, из второго утверждения леммы 5 вытекает, что $s l \mid\left(y_{2}-y_{1}\right)$, откуда, учитывая (3.25), получаем, что $s l \mid\left(k_{2}-k_{1}\right)$. Пусть $k_{2}=k_{1}+c s l$, где $c$ - целое число. Тогда из (3.30) следует, что $e /(s l) \mid c$, поэтому $e \mid c s l$ и $e \mid\left(k_{2}-k_{1}\right)$. Учитывая (3.25), получаем, что $e \mid\left(y_{2}-y_{1}\right)$, но это невозможно, так как $y_{2} \neq y_{1}$ и $\left|y_{2}-y_{1}\right|<d\left(a_{1}^{i}\right)-b\left(a_{1}^{i}\right)=d-b \leqslant e$. Таким образом, предположив, что $i_{1}=i_{2}=i$, мы пришли к противоречию.

Обозначим через $N_{3}$ количество различных троек чисел $(k, x, y)$, для которых $b_{x y}^{k}=1$, а через $N_{2}$ - количество различных пар $\left(u_{k}(x), v_{k}(y)\right)$. Очевидно, что $N_{3}=N \rho$. Из вышеизложенного вытекает, что различным тройкам $(k, x, y)$, для которых $b_{x y}^{k}=1$, соответствуют различные пары $\left(u_{k}(x), v_{k}(y)\right)$, поэтому $N_{2} \geqslant N_{3}$ и $N_{2} \geqslant N \rho$. Но из неравенств (3.23) следует, что $N_{2} \leqslant \rho N$, поэтому $N_{2}=\rho N$. Таким образом, для любой пары чисел $(u, v)$, где $1 \leqslant u \leqslant \rho, 1 \leqslant v \leqslant N$, существует ровно одна тройка $(k, x, y), 1 \leqslant k \leqslant N$, $1 \leqslant x \leqslant r, 1 \leqslant y \leqslant t$, для которой $b_{x, y}^{k}=1, u_{k}(x)=u$ и $v_{k}(y)=v$. Отсюда вытекает, что любая единица матрицы $D$ принадлежит ровно одной из усеченных подматриц $D^{1}, \ldots, D^{N}$.

3. Из п. 1, 2 вытекает, что $F$ - такое взаимно однозначное соответствие, при котором любая единица матрицы $D=E_{\rho \times N}$ принадлежит ровно одной из ее усеченных подматриц $D^{1}, \ldots, D^{N}$ и $D^{k} \cong B^{k} \cong A$ для любого $k=1, \ldots, N$. Значит, тождественная матрица $E_{\rho \times N}$ точно покрывается с помощью $N$ матриц $A$. Теперь оценим величину $N$. Ясно, что

$$
N=\operatorname{lcm}\left(e^{1}, \ldots, e^{m}\right)\left\lceil\frac{t}{\operatorname{lcm}\left(e^{1}, \ldots, e^{m}\right)}\right\rceil \leqslant t e^{1} \ldots e^{m} \leqslant t \prod_{i=1}^{m} s^{i} r^{i} \max _{1 \leqslant j \leqslant r^{i}} e\left(a_{j}^{i}\right) .
$$

Так как $m \leqslant r, e\left(a_{j}^{i}\right) \leqslant t$ и $s^{i} r^{i}<\left(d\left(a_{1}^{i}\right)-b\left(a_{1}^{i}\right)\right) / l^{i}+1$, то есть

$$
s^{i} r^{i} \leqslant \frac{d\left(a_{1}^{i}\right)-b\left(a_{1}^{i}\right)}{l^{i}} \leqslant t,
$$

отсюда следует, что $N \leqslant t \cdot t^{r} \cdot t^{r}=t^{2 r+1}$.

Теорема доказана.

Рассмотрим пример применения описанного в теореме 2 алгоритма точного покрытия тождественной матрищы. 
Пусть

$$
A=A_{r \times t}=\left(\begin{array}{cccc}
0 & 1 & 0 & 1 \\
1 & 0 & 1 & 1 \\
1 & 1 & 0 & 1
\end{array}\right)
$$

тогда $r=3, t=4, \rho=8$.

Поскольку агрегации

$$
\begin{aligned}
& g^{1}=\operatorname{agr}_{1}^{2}(0101)=(01111) \\
& g^{2}=\operatorname{agr}_{2}^{1}((1101),(1011))=(111111)
\end{aligned}
$$

представляют собой сплошные $(0,1)$-строки, строки матрицы $A$ разбиваются на две агрегируемые группы $R^{1}, R^{2}$, где группа $R^{1}$ содержит первую, а группа $R^{2}$ - третью и вторую строки. Отсюда следует, что матрица $A$ является агрегируемой. Обозначим через $a_{1}^{1}$ первую, через $a_{1}^{2}$ третью, а через $a_{2}^{2}$ вторую строку матрицы $A$. Пусть группа $R^{i}$, $i=1,2$, содержит $r^{i}$ строк и является агрегируемой с параметрами $s^{i}, l^{i}$. Тогда

$$
r^{1}=1, \quad s^{1}=2, \quad l^{1}=1, \quad r^{2}=2, \quad s^{2}=1, \quad l^{2}=2 .
$$

Пусть строка матрищы $A$ с номером $x, x=1,2,3$, принадлежит группе $R^{i(x)}$ и имеет в ней номер $j(x)$. Очевидно, что

$$
i(1)=1, \quad j(1)=1, \quad i(2)=2, \quad j(2)=2, \quad i(3)=2, \quad j(3)=1 .
$$

Пусть агрегация $g^{i}, i=1,2$, содержит $e^{i}$ единиц. Тогда $e^{1}=4$ и $e^{2}=6$. Положим

$$
N=\operatorname{lcm}\left(e^{1}, e^{2}\right)\left\lceil t / \operatorname{lcm}\left(e^{1}, e^{2}\right)\right\rceil=12 .
$$

Построим точное покрытие тождественной матрицы $E_{8 \times 12}$ с помощью $N$ матриц $A$.

Через $B^{k}=\left\|b_{x y}^{k}\right\|, k=1, \ldots, 12$, обозначим $k$-ю матрицу $A$, участвующую в покрытин. Для каждого $k=1, \ldots, 12$ матрице $B^{k}$ поставим в соответствие усеченную подматрицу $D^{k}=\left\|d_{x y}^{k}\right\|$ матрицы $E_{8 \times 12}$, расположенную в строках с номерами $u_{k}(1), \ldots, u_{k}(3)$ и столбцах с номерами $v_{k}(1), \ldots, v_{k}(4)$, так, чтобы $b_{x y}^{k}=d_{x y}^{k}$, где величины $u_{k}(x), v_{k}(y)$ определяются с помощью равенств (3.19)-(3.21). Очевидно, что тогда

$$
\begin{aligned}
u_{k}(1) & =w_{k}(1)+1=\lfloor(k-1) / 2\rfloor \bmod 2+(k-1) \bmod 1+1 \\
& =\lfloor(k-1) / 2\rfloor \bmod 2+1, \\
u_{k}(2) & =e^{1} / s^{1}+w_{k}(2)+1=2+(k-3) \bmod 6+1=3+(k-3) \bmod 6, \\
u_{k}(3) & =e^{1} / s^{1}+w_{k}(3)+1=2+(k-1) \bmod 6+1=3+(k-1) \cdot \bmod 6, \\
v_{k}(1) & =(k-1) \bmod 12+1 \\
v_{k}(2) & =k \bmod 12+1 \\
v_{k}(3) & =(k+1) \bmod 12+1 \\
v_{k}(4) & =(k+2) \bmod 12+1
\end{aligned}
$$

Сопоставим тождественной матрице $E_{8 \times 12}$ таблицу $T$ из восьми строк и двенадцати столбцов, в которой на пересечении строки с номером $u, u=1, \ldots, 8$, и столбца с номером $v, v=1, \ldots, 12$, стоит такое число $k$, что $u=u_{k}(x), v=v_{k}(y)$ и $b_{x y}^{k}=1$. 
Тогда таблица $T$ будет выглядеть следующим образом:

$$
T=\left(\begin{array}{cccccccccccc}
10 & 1 & 2 & 1 & 2 & 5 & 6 & 5 & 6 & 9 & 10 & 9 \\
12 & 11 & 12 & 3 & 4 & 3 & 4 & 7 & 8 & 7 & 8 & 11 \\
1 & 1 & 3 & 1 & 3 & 3 & 7 & 7 & 9 & 7 & 9 & 9 \\
10 & 2 & 2 & 4 & 2 & 4 & 4 & 8 & 8 & 10 & 8 & 10 \\
11 & 11 & 3 & 3 & 5 & 3 & 5 & 5 & 9 & 9 & 11 & 9 \\
10 & 12 & 12 & 4 & 4 & 6 & 4 & 6 & 6 & 10 & 10 & 12 \\
1 & 11 & 1 & 1 & 5 & 5 & 7 & 5 & 7 & 7 & 11 & 11 \\
12 & 2 & 12 & 2 & 2 & 6 & 6 & 8 & 6 & 8 & 8 & 12
\end{array}\right)
$$

\section{4. Алгоритм построения точного покрытия графа для слабо плотных базисов специального вида}

Пусть двудольный граф $H \in \mathscr{H}$ имеет сокращенную матрицу смежности $A$, и пусть существует такая агрегируемая матрища $A^{\prime}$, что $A \cong A^{\prime}$. Тогда граф $H$ называется агрегируемым.

Очевидно, что тривиальный граф, содержащийся в любом базисе, является агрегируемым.

Теорема 3. Для любого вещественного $\varepsilon, 0<\varepsilon<1$, существует алгоритм $A_{\varepsilon}$, который для любого слабо плотного базиса $B$ с весом $\rho$, содержащего агрегируемый двудольный граф с $\rho$ ребрами, и любого графа $G \in \mathscr{G}$ с $p$ вериинами и q ребрами строит точное покрытие графа $G$ в базисе $B$ со сложностью $L_{\varepsilon}(q, p, \rho)$, где

$$
L_{\varepsilon}(q, p, \rho) \leqslant \frac{q}{\rho}+\frac{\rho p^{2}}{\varepsilon \log _{2} p}+\frac{\varepsilon}{2} p \log _{2} p+2 \rho^{2 \rho+1} p^{1+\varepsilon}+\frac{p}{2}+\varepsilon O\left(\left(\log _{2} p\right)^{2}\right) .
$$

Доказательство. Пусть $\varepsilon-$ произвольное вещественное число, $0<\varepsilon<1$, и пусть слабо плотный базис $B$ с весом $\rho$ содержит агрегируемый двудольный граф $H=H_{r, t} \in \mathscr{H}$ с $\rho$ ребрами, $1 \leqslant \rho \leqslant r t$, имеющий сокращенную матрицу смежности $A=A_{r \times t}$, и тривиальный граф $H^{0}$, имеющий сокращенную матрицу смежности $A^{0}=A_{1 \times 1}^{0}=(1)$. Пусть $A^{\prime}$ - такая агрегируемая матрица, что $A^{\prime} \cong A$ и $A^{\prime}$ является сокращенной матрицей смежности двудольного графа $H^{\prime}$ с $\rho$ ребрами. Тогда по лемме $1 H^{\prime} \cong H$, а матрица $A^{\prime}$ имеет размер $r \times t$ или $t \times r$. Поскольку $H \in \mathscr{H}$, матрица $A^{\prime}$ не содержит нулевых строк.

Пусть $G \in \mathscr{G}$ - произвольный граф, $q(G)=q, p(G)=p$, и пусть $M=M_{p \times p}$ - матрица смежности графа $G$. Построим точное покрытие верхнего сокращенного треугольника матрицы $M$ матрицами $A^{\prime}$ и $A^{0}$.

Положим $h=\left\lceil\log _{2} p^{\varepsilon}\right\rceil$. Очевидно, что $1 \leqslant h \leqslant p$.

Через $P_{i}, i=1, \ldots,\lceil p / h\rceil-1$, обозначим подматрицу матрицы $M$, расположенную на пересечении строк с номерами $(i-1) h+1, \ldots, i h$ и столбцов с номерами $i h+1, \ldots, p$. Назовем эту подматрицу $i$-м прямоугольником. Обозначим через $Q_{i}, i=1, \ldots,\lceil p / h\rceil$, подматрицу матрицы $M$, расположенную на пересечении строк с номерами $(i-1) h+1, \ldots, \min (i h, p)$ и столбцов с номерами $(i-1) h+1, \ldots, \min (i h, p)$. Назовем эту подматрицу $i$-м квадратом. Очевидно, что прямоугольник $P_{i}$ содержит $h$ строк и $p-i h$ столбцов, а квадрат $Q_{i}$ - не более $h$ строк и не более $h$ столбцов.

Пусть $e_{i}$ - число единиц, а $c_{i}$ - число различных столбцов в прямоугольнике $P_{i}$. Тогда $1 \leqslant c_{i} \leqslant 2^{h}$. Разобьем столбцы прямоугольника $P_{i}$ на $c_{i}$ классов эквивалентности $R_{1}^{i}, \ldots, R_{c_{i}}^{i}$, понимая под эквивалентностью равенство. Очевидно, что для любого 
$i=1, \ldots,\lceil p / h\rceil-1$ и любого $j=1, \ldots, c_{i}$ группа столбцов $R_{j}^{i}$ высоты $h$ представляет собой подматрицу прямоугольника $P_{i}$, в которой любая строка либо единичная, либо нулевая, при этом каждый элемент прямоугольника $P_{i}$ принадлежит ровно одной из подматрищ $R_{1}^{i}, \ldots, R_{c_{i}}^{i}$. Пусть матрища $R_{j}^{i}$ содержит $m_{j}^{i}$ столбцов, $h_{j}^{i}$ единичных строк и $e_{j}^{i}=m_{j}^{i} h_{j}^{i}$ единиц.

Пусть $N \leqslant \max \left(t^{2 r+1}, r^{2 t+1}\right)$ - такое натуральное число, что с помощью $N$ матриц $A^{\prime}$ можно точно покрыть тождественную матрицу $E_{\rho \times N}$ (по теореме 2 такое $N$ существует). Пусть $I_{j}^{i}$ - матрица, состоящая из первых $\left\lfloor h_{j}^{i} / \rho\right\rfloor \rho$ единичных строк и первых $\left\lfloor m_{j}^{i} / N\right\rfloor N$ столбцов матрицы $R_{j}^{i}$.

Для любого $i=1, \ldots,\lceil p / h\rceil-1$ и любого $j=1, \ldots, c_{i}$ разобьем матрицу $I_{j}^{i}$ на $l(i, j)=\left\lfloor h_{j}^{i} / \rho\right\rfloor\left\lfloor m_{j}^{i} / N\right\rfloor$ непересекающихся тождественных подматриц, содержащих по $\rho$ строк и $N$ столбцов. Каждую из них точно покроем с помощью $N$ матриц $A^{\prime}$ описанным в теореме 2 способом. Единицы матрищы $R_{j}^{i}$, не принадлежащие матрице $I_{j}^{i}$, точно покроем с помощью $l_{0}(i, j) \leqslant \rho m_{j}^{i}+N h_{j}^{i}$ матриц $A^{0}$. Для любого $i=1, \ldots,\lceil p / h\rceil$ покроем верхний сокращенный треугольник квадрата $Q_{i}$ с помощью $l_{1}(i)=(1 / 2) e\left(Q_{i}\right)$ матриц $A^{0}$.

Таким образом мы построим точное покрытие матрицы $M$ с помощью некоторого количества матриц $A^{\prime}$ и $A^{0}$. Пусть $L$ - сложность этого покрытия, $L_{A^{\prime}}$ (соответственно $L_{A^{0}}$ ) - число участвующих в нем матриц $A^{\prime}$ (соответственно $A^{0}$ ), тогда $L=L_{A^{\prime}}+L_{A^{0}}$. Оценим величины $L_{A^{\prime}}$ и $L_{A^{0}}$. Нетрудно видетъ, что

$$
\begin{aligned}
L_{A^{\prime}} & =\sum_{i=1}^{\lceil p / h\rceil-1} \sum_{j=1}^{c_{i}} l(i, j) N=N \sum_{i=1}^{\lceil p / h\rceil-1} \sum_{j=1}^{c_{i}}\left\lfloor\frac{h_{j}^{i}}{\rho}\right\rfloor\left\lfloor\frac{m_{j}^{i}}{N}\right\rfloor \\
& \leqslant \frac{N}{\rho N} \sum_{i=1}^{\lceil p / h\rceil-1} \sum_{j=1}^{c_{i}} e_{j}^{i}=\frac{1}{\rho} \sum_{i=1}^{\lceil p / h\rceil-1} e_{i} \leqslant \frac{q}{\rho} . \\
L_{A^{0}} & =\sum_{i=1}^{\lceil p / h\rceil-1} \sum_{j=1}^{c_{i}} l_{0}(i, j)+\sum_{i=1}^{\lceil p / h\rceil} l_{1}(i) \leqslant \sum_{i=1}^{\lceil p / h\rceil-1}\left(\rho \sum_{j=1}^{c_{i}} m_{j}^{i}+N \sum_{j=1}^{c_{i}} h_{j}^{i}\right)+\sum_{i=1}^{\lceil p / h\rceil} \frac{h^{2}}{2} \\
& \leqslant \frac{p}{h}\left(\rho p+N 2^{h} h\right)+(p / h+1) \frac{h^{2}}{2}=\rho \frac{p^{2}}{h}+N p 2^{h}+\frac{p h}{2}+\frac{h^{2}}{2} .
\end{aligned}
$$

Так как $N \leqslant \max \left(t^{2 r+1}, r^{2 t+1}\right) \leqslant \rho^{2 \rho+1}$ и $h=\left\lceil\log _{2} p^{\varepsilon}\right\rceil=\left\lceil\varepsilon \log _{2} p\right\rceil$, справедливы оценки

$$
\begin{aligned}
L & =L_{A^{\prime}}+L_{A^{0}} \leqslant \frac{q}{\rho}+\frac{\rho p^{2}}{\varepsilon \log _{2} p}+\rho^{2 \rho+1} p 2^{\left\lceil\log _{2} p^{\varepsilon}\right\rceil}+\frac{1}{2} p\left\lceil\log _{2} p^{\varepsilon}\right\rceil+\frac{1}{2}\left(\left\lceil\log _{2} p^{\varepsilon}\right\rceil\right)^{2} \\
& \leqslant \frac{q}{\rho}+\frac{\rho p^{2}}{\varepsilon \log _{2} p}+2 \rho^{2 \rho+1} p^{1+\varepsilon}+\frac{\varepsilon}{2} p \log _{2} p+\frac{p}{2}+\varepsilon O\left(\left(\log _{2} p\right)^{2}\right),
\end{aligned}
$$

то есть для сложности $L$ выполняется неравенство (4.1). По теореме 1 построенному нами точному покрытию матрицы $M$ матрицами $A^{\prime}$ и $A^{0}$ соответствует точное покрытие графа $G$ графами $H^{\prime}$ и $H^{0}$ той же сложности. Поскольку $H^{\prime} \cong H$, применив описанный алгоритм, можно построить точное покрытие графа $G$ графами $H$ и $H^{0}$ со сложностью $L$, для которой выполняется неравенство (4.1). Теорема доказана. 
Следствие 2. Если базис В с весом $\rho$ является слабо плотным и содержст агрегируемый двудольный граф с $\rho$ ребрами, то

$$
l_{B}(q, p) \leqslant \frac{q}{\rho}+O\left(\frac{p^{2}}{\log _{2} p}\right) .
$$

\section{5. Алгоритм построения точного покрытия графа для произвольного слабо плотного базиса}

Пусть $A^{1}=\left\|a_{i j}^{1}\right\|$ и $A^{2}=\left\|a_{i j}^{2}\right\|$ - матрицы размера $r \times t, r, t \geqslant 1$. Тогда матрица $A=\left\|a_{i j}^{1}+a_{i j}^{2}\right\|$ размера $r \times t$ называется суммой матриц $A^{1}$ и $A^{2}$ и обозначается $A^{1}+A^{2}$.

Пусть $A=A_{r \times t}=\left\|a_{i j}\right\|, r, t \geqslant 1,-(0,1)$-матрица, $m, n-$ натуральные числа. Тогда $(m, n)$-разверткой матрицы $A$ называется $(0,1)$-матрица $\mathscr{R}^{m, n}(A)=\left\|r_{x y}\right\|$, содержащая $m r$ строк и $n t$ столбцов, в которой

$$
r_{x y}=a_{(x-1)} \bmod r+1,(y-1) \bmod t+1, \quad x=1, \ldots, m r, \quad y=1, \ldots, n t .
$$

Очевидно, что $e\left(\mathscr{R}^{m, n}(A)\right)=m n e(A), z\left(\mathscr{R}^{m, n}(A)\right)=m n z(A)$.

Пример 1. Пусть

$$
A=\left(\begin{array}{ll}
1 & 0 \\
1 & 1
\end{array}\right), \quad m=2, \quad n=2
$$

Тогда

$$
\mathscr{R}^{2,2}=\left(\begin{array}{llll}
1 & 0 & 1 & 0 \\
1 & 1 & 1 & 1 \\
1 & 0 & 1 & 0 \\
1 & 1 & 1 & 1
\end{array}\right) .
$$

Пусть $A=A_{r \times t}=\left\|a_{i j}\right\|, r, t \geqslant 1,-(0,1)$-матрица, $m, n, f, g$ - натуральные числа, $1 \leqslant f \leqslant m, 1 \leqslant g \leqslant n$. Тогда $(m, n)$-распределением матрицы $A$ по элементу $(f, g)$ называется $(0,1)$-матрица $\mathscr{D}^{m, n}(A, f, g)=\left\|d_{x y}\right\|$, содержащая $m r$ строк и $n t$ столбцов, в которой $d_{x y}=a_{i j}$, если $x=f+(i-1) m, y=g+(j-1) n$, и $d_{x y}=0$ в противном случае, то есть $d_{x y}=a_{1+(x-f) / m, 1+(y-g) / n}$, если $m|(x-f), n|(y-g)$, и $d_{x y}=0$ в противном случае, $x=1, \ldots, m r, y=1, \ldots, n t$. Ясно, что $e\left(\mathscr{D}^{m, n}(A, f, g)\right)=e(A)$.

Пример 2. Пусть

$$
A=\left(\begin{array}{ll}
1 & 0 \\
1 & 1
\end{array}\right), \quad m=2, \quad n=2, \quad f=1, \quad g=2
$$

Тогда

$$
\mathscr{D}^{2,2}(A, 1,2)=\left(\begin{array}{llll}
0 & 1 & 0 & 0 \\
0 & 0 & 0 & 0 \\
0 & 1 & 0 & 1 \\
0 & 0 & 0 & 0
\end{array}\right) .
$$


Пусть $A=A_{r \times t}=\left\|a_{i j}\right\|, r, t \geqslant 1,-(0,1)$-матрица, $N$ - натуральное число. Тогда $N$-укладкой матрицы $A$ называется матрица $U^{N}(A)$, содержащая $r^{N}$ строк и $t^{N}$ столбцов, определяемая следующим образом. Если $N=1$, то $U^{N}(A)=A$. Если $N>1$ и $U^{N-1}(A)-$ $(N-1)$-укладка матрищы $A$, содержащая $r^{N-1}$ строк, $t^{N-1}$ столбцов и $z_{N-1}=z\left(U^{N-1}(A)\right)$ нулей в позициях $\left(f_{1}^{N-1}, g_{1}^{N-1}\right), \ldots,\left(f_{z_{N-1}}^{N-1}, g_{z_{N-1}}^{N-1}\right)$, то

$$
U^{N}(A)=\mathscr{R}^{r, t}\left(U^{N-1}(A)\right)+\sum_{q=1}^{z_{N-1}} \mathscr{D}^{r^{N-1}, t^{N-1}}\left(A, f_{q}^{N-1}, g_{q}^{N-1}\right)
$$

Пример 3. Пусть

$$
A=\left(\begin{array}{ll}
1 & 0 \\
1 & 1
\end{array}\right), \quad N=2
$$

Тогда

$$
U^{2}(A)=\mathscr{R}^{2,2}(A)+\mathscr{D}^{2,2}(A, 1,2)=\left(\begin{array}{llll}
1 & 1 & 1 & 0 \\
1 & 1 & 1 & 1 \\
1 & 1 & 1 & 1 \\
1 & 1 & 1 & 1
\end{array}\right) .
$$

Лемма 6. Пусть $A=A_{r \times t}=\left\|a_{i j}\right\|, r, t \geqslant 1,-(0,1)$-матрича. Тогда для любого натурального $N$ укладка $U^{N}(A)$ является $(0,1)$-матричей $и z\left(U^{N}(A)\right)=(z(A))^{N}$.

Доказательство. Докажем лемму по индукции.

Пусть $N=1$. Тогда $U^{N}(A)=A$, и справедливость утверждения леммы очевидна.

Пусть $N>1$, и пусть для $(N-1)$-укладки $U^{N-1}(A)$ утверждение леммы выполнено. Введем обозначения $U^{N-1}(A)=U^{N-1}, U^{N}(A)=U^{N}, z(A)=z$. Тогда по предположению индукции матрица $U^{N-1}$ является $(0,1)$-матрицей, при этом $z\left(U^{N-1}\right)=z^{N-1}$.

Пусть нули матрицы $U^{N-1}$ расположены в позициях $\left(f_{1}, g_{1}\right), \ldots,\left(f_{z^{N-1}}, g_{z^{N-1}}\right)$. Тогда по определению $N$-укладки

$$
U^{N}=\mathscr{R}^{r, t}\left(U^{N-1}\right)+\sum_{q=1}^{z^{N-1}} \mathscr{D}^{r^{N-1}, t^{N-1}}\left(A, f_{q}, g_{q}\right)
$$

Пусть $U^{N}=\left\|u_{x y}^{N}\right\|, U^{N-1}=\left\|u_{x y}^{N-1}\right\|, A=\left\|a_{i j}\right\|$. Тогда из (5.2) и введенных определений следует, что для любых $x, y$ таких, что $1 \leqslant x \leqslant r^{N}, 1 \leqslant y \leqslant t^{N}$, выполнено равенство

$$
u_{x, y}^{N}=u_{(x-1)}^{N-1} \bmod r^{N-1}+1,(y-1) \bmod t^{N-1}+1+U
$$

где

$$
U=\sum_{q=1, r^{N-1}\left|\left(x-f_{q}\right), t^{N-1}\right|\left(y-g_{q}\right)}^{z^{N-1}} a_{1+\left(x-f_{q}\right) / r^{N-1}, 1+\left(y-g_{q}\right) / t^{N-1}}
$$

По предположению индукции укладка $U^{N-1}$ является $(0,1)$-матрицей. Значит, возможны два случая.

В первом случае $u_{(x-1) \bmod r^{N-1}+1}^{N-1},(y-1) \bmod t^{N-1}+1=0$. Тогда существует единственное число $q, 1 \leqslant q \leqslant z^{N-1}$, такое, что $f_{q}=(x-1) \bmod r^{N-1}+1$, 
$g_{q}=(y-1) \bmod t^{N-1}+1$, то есть $r^{N-1} \mid\left(x-f_{q}\right)$ и $t^{N-1} \mid\left(y-g_{q}\right)$. Учитывая (5.3), получаем, что в этом случае

$$
u_{x, y}^{N}=0+a_{1+\left(x-f_{q}\right) / r^{N-1}, 1+\left(y-g_{q}\right) / t^{N-1},}
$$

значит, $u_{x, y}^{N}$ равно нулю или единище.

Во втором случае $u_{(x-1)}^{N-1} \bmod r^{N-1}+1,(y-1) \bmod t^{N-1}+1=1$. Тогда не существует такого числа $q, 1 \leqslant q \leqslant z^{N-1}$, что

$$
f_{q}=(x-1) \bmod r^{N-1}+1, \quad g_{q}=(y-1) \bmod t^{N-1}+1,
$$

то есть $r^{N-1} \mid\left(x-f_{q}\right)$ и $t^{N-1} \mid\left(y-g_{q}\right)$. Отсюда и из (5.3) следует, что

$$
u_{x, y}^{N}=u_{(x-1)}^{N-1} \bmod r^{N-1}+1,(y-1) \bmod t^{N-1}+1+0=1 .
$$

Таким образом, матрица $U^{N}$ является $(0,1)$-матрицей. Покажем, что $z\left(U^{N}\right)=z^{N}$. Очевидно, что

$$
\begin{aligned}
e\left(U^{N}\right) & =e\left(\mathscr{R}^{r, t}\left(U^{N-1}\right)\right)+\sum_{q=1}^{z^{N-1}} e\left(\mathscr{D}^{r^{N-1}, t^{N-1}}\left(A, f_{q}, g_{q}\right)\right) \\
& =r t e\left(U^{N-1}\right)+\sum_{q=1}^{z^{N-1}} e(A)=r t\left(r^{N-1} t^{N-1}-z\left(U^{N-1}\right)\right)+z^{N-1}(r t-z(A)) \\
& =r^{N} t^{N}-r t z^{N-1}+z^{N-1} r t-z^{N}=r^{N} t^{N}-z^{N} .
\end{aligned}
$$

Значит, $z\left(U^{N}\right)=r^{N} t^{N}-e\left(U^{N}\right)=z^{N}$, что завершает доказательство леммы.

Пусть $A=A_{r \times t}=\left\|a_{i j}\right\|, r, t \geqslant 1,-(0,1)$-матрица, $N$ - натуральное число. Величина

$$
\frac{e\left(U^{N}(A)\right)}{e(A)}=\frac{r^{N} t^{N}-(z(A))^{N}}{r t-z(A)}
$$

называется мощностью $N$-укладки матрицы $A$ и обозначается $\mathscr{P}^{N}(A)$.

Теорема 4. Пусть $A=A_{r \times t}=\left\|a_{i j}\right\|, r, t \geqslant 1,-(0,1)$-матрица, $A^{0}$ - одноэлементная матрича, состоящая из единицы, $N$ - натуральное число. Тогда тождественная матрича $E_{r^{N} \times t^{N}}$ точно покрывается с помощью $\mathscr{P}^{N}(A)$ матрич $A u(z(A))^{N}$ матрии $A^{0}$.

Доказательство. Из леммы 6 и введенных определений следует, что в матрице $U^{N}(A)$ можно выделить $e\left(U^{N}(A)\right) / e(A)$ усеченных подматриц вида $A$, не пересекающихся по единицам и покрывающих все единицы матрицы $U^{N}(A)$. Значит, укладка $U^{N}(A)$ точно покрывается с помощью $e\left(U^{N}(A)\right) / e(A)=\mathscr{P}^{N}(A)$ матриц $A$. Очевидно, что тождественная матрица $E_{r^{N} \times t^{N}}$ содержит усеченную подматрицу вида $U^{N}(A)$. При этом те единицы матрицы $E_{r^{N} \times t^{N}}$, которые соответствуют единицам укладки $U^{N}(A)$, точно покрываются с помощью $\mathscr{P}^{N}(A)$ матриц $A$. Оставшиеся $(z(A))^{N}$ единиц матрищы $E_{r^{N} \times t^{N}}$ точно покрываются с помощью $(z(A))^{N}$ матриц $A^{0}$. В результате получается точное покрытие матрищы $E_{r^{N} \times t^{N}}$, в котором участвуют $\mathscr{P}^{N}(A)$ матриц $A$ и $(z(A))^{N}$ матриц $A^{0}$. Теорема доказана. 
Теорема 5. Для любых вещественных чисел $\beta$ и $\varepsilon$ таких, что $0<\varepsilon<\beta<1$, существует алгоритм $A_{\beta, \varepsilon}$, который для любого слабо плотного базиса В с весом $\rho$ и любого графа $G \in \mathscr{G}_{c}$ р вериинами и q ребрами строчт точное покрытие графа $G$ в базисе $B$ со сложностью $L_{\beta, \varepsilon}(q, p, \rho)$, где

$$
\begin{aligned}
L_{\beta, \varepsilon}(q, p, \rho) \leqslant \frac{q}{\rho}+ & \frac{(1+\rho) p^{2}}{\left(\varepsilon \log _{2} p\right)^{\left(1-\log _{1+\rho} \rho\right)(1-\beta)}}+\frac{p^{2}+2 p^{1+\varepsilon}}{\left(\varepsilon \log _{2} p\right)^{\beta}} \\
& +2 p^{1+\varepsilon}\left(\varepsilon \log _{2} p\right)^{(1-\beta)}+\frac{p \varepsilon \log _{2} p}{2}+\frac{p}{2}+\varepsilon O\left(\left(\log _{2} p\right)^{2}\right) .
\end{aligned}
$$

Доказательство. Пусть $\beta, \varepsilon-$ произвольные вещественные числа, $0<\varepsilon<\beta<1$. Известно (см. [4]), что базис с весом $\rho$ является слабо плотным тогда и только тогда, когда он содержит двудольный граф с $\rho$ ребрами. Пусть слабо плотный базис $B$ с весом $\rho$ содержит двудольный граф $H=H_{r, t}$ с $\rho$ ребрами, $1 \leqslant \rho \leqslant r t$, имеющий сокращенную матрицу смежности $A=A_{r \times t}$, и тривиальный граф $H^{0}$, имеющий сокращенную матрицу смежности $A^{0}=A_{1 \times 1}^{0}=(1)$. Положим $z=z(A)$.

Пусть $G \in \mathscr{G}$ - произвольный граф с $p$ вершинами и $q$ ребрами, имеющий матрицу смежности $M=M_{p \times p}$. Построим точное покрытие верхнего сокращенного треугольника матрицы $M$ матрицами $A$ и $A^{0}$.

Положим $h=\left\lceil\log _{2} p^{\varepsilon}\right\rceil$. Очевидно, что $1 \leqslant h \leqslant p$.

Через $P_{i}, i=1, \ldots,\lceil p / h\rceil-1$, обозначим подматрицу матрицы $M$, расположенную на пересечении строк с номерами $(i-1) h+1, \ldots, i h$ и столбцов с номерами $i h+1, \ldots, p$. Назовем эту подматрицу $i$-м прямоугольником. Обозначим через $Q_{i}, i=1, \ldots,\lceil p / h\rceil$, подматрицу матрицы $M$, расположенную на пересечении строк с номерами $(i-1) h+1, \ldots, \min (i h, p)$ и столбцов с номерами $(i-1) h+1, \ldots, \min (i h, p)$. Назовем эту подматрицу $i$-м квадратом. Очевидно, что прямоугольник $P_{i}$ содержит $h$ строк и $p-i h$ столбцов, а квадрат $Q_{i}-$ не более $h$ строк и не более $h$ столбцов.

Пусть $e_{i}$ - число единиц, а $c_{i}$ - число различных столбцов в прямоугольнике $P_{i}$. Тогда $1 \leqslant c_{i} \leqslant 2^{h}$. Разобьем столбцы прямоугольника $P_{i}$ на $c_{i}$ классов эквивалентности $R_{1}^{i}, \ldots, R_{c_{i}}^{i}$, понимая под эквивалентностью равенство. Очевидно, что для любого $i=1, \ldots,\lceil p / h\rceil-1$ и любого $j=1, \ldots, c_{i}$ группа столбцов $R_{j}^{i}$ высоты $h$ предсгавляет собой подматрицу прямоугольника $P_{i}$, в которой любая строка либо единичная, либо нулевая, при этом каждый элемент прямоугольника $P_{i}$ принадлежит ровно одной из подматриц $R_{1}^{i}, \ldots, R_{c_{i}}^{i}$. Пусть матрица $R_{j}^{i}$ содержит $m_{j}^{i}$ столбцов, $h_{j}^{i}$ единичных строк и $e_{j}^{i}=m_{j}^{i} h_{j}^{i}$ единиц.

Положим $N_{j}^{i}=\left\lfloor(1-\beta) \log _{r t} h_{j}^{i}\right\rfloor, i=1, \ldots,\lceil p / h\rceil-1, j=1, \ldots, c_{i}$. Тогда

$$
(r t)^{N_{j}^{i}} \leqslant\left(h_{j}^{i}\right)^{1-\beta}<(r t)^{N_{j}^{i}+1}
$$

Пусть $I_{j}^{i}$ - матрица, состоящая из первых $\left\lfloor h_{j}^{i} / r^{N_{j}^{i}}\right\rfloor r^{N_{j}^{i}}$ единичных строк и первых $\left\lfloor m_{j}^{i} / t^{N_{j}^{i}}\right\rfloor t^{N_{j}}$ столбцов матрицы $R_{j}^{i}$. Для любого $i=1, \ldots,\lceil p / h\rceil-1$ и любого $j=1, \ldots, c_{i}$ разобьем матрицу $I_{j}^{i}$ на $l(i, j)=\left\lfloor h_{j}^{i} / r^{N_{j}^{i}}\right\rfloor\left\lfloor m_{j}^{i} / t N_{j}^{i}\right\rfloor$ непересекающихся тождественных подматриц вида $E_{r}{ }_{N_{x t}^{i}} N_{j}^{i}$, каждую из которых точно покроем с помощью $l_{1}(i, j)=\mathscr{P}^{N_{j}^{i}}(A)$ матриц $A$ и $l_{2}(i, j)=z^{N_{j}^{i}}$ матриц $A^{0}$ согласно теореме 4. Единицы матрицы $R_{j}^{i}$, не принадлежащие матрице $I_{j}^{i}$, точно покроем с помощью $l_{0}(i, j) \leqslant r^{N_{j}^{i}} m_{j}^{i}+t N_{j}^{i} h_{j}^{i}$ 
матриц $A^{0}$. Для любого $i=1, \ldots,\lceil p / h\rceil$ точно покроем верхний сокращенный треугольник квадрата $Q^{i}$ с помощью $l_{3}(i)=(1 / 2) e\left(Q_{i}\right)$ матрищ $A^{0}$.

Таким образом мы построим точное покрытие матрицы $M$ матрицами $A$ и $A^{0}$. Пусть $L$ - сложность этого покрытия, $L_{A}$ (соответственно $\left.L_{A^{0}}\right)$ - число участвующих в нем матрищ $A$ (соответственно $A^{0}$ ), тогда $L=L_{A}+L_{A^{0}}$. Оценим величины $L_{A}$ и $L_{A^{0}}$. Нетрудно видеть, что

$$
\begin{aligned}
& L_{A}=\sum_{i=1}^{\lceil p / h\rceil-1} \sum_{j=1}^{c_{i}} l(i, j) l_{1}(i, j)=\sum_{i=1}^{\lceil p / h\rceil-1} \sum_{j=1}^{c_{i}}\left\lfloor h_{j}^{i} / r^{N_{j}^{i}}\right\rfloor\left\lfloor m_{j}^{i} / t^{N_{j}^{i}}\right\rfloor \mathscr{P}^{N_{j}^{i}}(A) \\
& \leqslant \sum_{i=1}^{\lceil p / h\rceil-1} \sum_{j=1}^{c_{i}} \frac{\left(r^{\left.N_{j}^{i} t^{N_{j}^{i}}-z^{N_{j}^{i}}\right)}\right.}{(r t-z) r^{N_{j}^{i} N_{j}^{i}}} h_{j}^{i} m_{j}^{i}=\frac{1}{r t-z} \sum_{i=1}^{\lceil p / h\rceil-1} \sum_{j=1}^{c_{i}}\left(1-\frac{z^{N_{j}^{i}}}{r^{N_{j}^{i} N_{j}^{i}}}\right) h_{j}^{i} m_{j}^{i} \\
& \leqslant \frac{1}{\rho} \sum_{i=1}^{\lceil p / h\rceil-1} \sum_{j=1}^{c_{i}} e_{j}^{i}=\frac{1}{\rho} \sum_{i=1}^{\lceil p / h\rceil-1} e_{i} \leqslant \frac{q}{\rho} \\
& L_{A^{0}}=\sum_{i=1}^{\lceil p / h\rceil-1} \sum_{j=1}^{c_{i}}\left(l(i, j) l_{2}(i, j)+l_{0}(i, j)\right)+\sum_{i=1}^{\lceil p / h\rceil} l_{3}(i) \\
& \leqslant \sum_{i=1}^{\lceil p / h\rceil-1} \sum_{j=1}^{c_{i}} \frac{h_{j}^{i} m_{j}^{i}}{r^{i} N_{j}^{i}} z^{N_{j}^{i}}+\sum_{i=1}^{\lceil p / h\rceil-1} \sum_{j=1}^{c_{i}}\left(r^{N_{j}^{i}} m_{j}^{i}+t^{N_{j}^{i}} h_{j}^{i}\right)+\sum_{i=1}^{\lceil p / h\rceil} \frac{h^{2}}{2} .
\end{aligned}
$$

Положим

$$
S=\sum_{i=1}^{\lceil p / h\rceil-1} \sum_{j=1}^{c_{i}} \frac{h_{j}^{i} m_{j}^{i}}{r^{N_{j}^{i} t^{N_{j}^{i}} z^{N_{j}^{i}}} .}
$$

Тогда, поскольку $(r t)^{N_{j}^{i}} \leqslant\left(h_{j}^{i}\right)^{1-\beta}$ и $h_{j}^{i} \leqslant h$, справедливы оценки

$$
\begin{aligned}
L_{A^{0}} & \leqslant S+\sum_{i=1}^{\lceil p / h\rceil-1} \sum_{j=1}^{c_{i}}\left(\left(h_{j}^{i}\right)^{1-\beta} m_{j}^{i}+\left(h_{j}^{i}\right)^{1-\beta} h_{j}^{i}\right)+(p / h+1) \frac{h^{2}}{2} \\
& \leqslant S+h^{1-\beta} \sum_{i=1}^{\lceil p / h\rceil-1}\left(\sum_{j=1}^{c_{i}} m_{j}^{i}+\sum_{j=1}^{c_{i}} h_{j}^{i}\right)+(p / h+1) \frac{h^{2}}{2} \\
& \leqslant S+h^{1-\beta} \sum_{i=1}^{\lceil p / h\rceil-1}\left(p+2^{h} h\right)+(p / h+1) \frac{h^{2}}{2} \\
& \leqslant S+h^{1-\beta} p / h\left(p+2^{h} h\right)+(p / h+1) \frac{h^{2}}{2} \\
& =S+\frac{p^{2}}{h^{\beta}}+2^{h} h^{1-\beta} p+\frac{p h}{2}+\frac{h^{2}}{2} .
\end{aligned}
$$

Теперь оценим величину $S$. Рассмотрим два случая.

Если $z=0$, то, очевидно, что $S=0$.

Пусть $z>0$. Положим $\alpha=\log _{r t} z=\log _{z+\rho} z$, тогда $0 \leqslant \alpha<1$ и $z=(r t)^{\alpha}$. Из неравенств (5.5) вытекает, что $(r t)^{N_{j}^{i}}>\left(h_{j}^{i}\right)^{1-\beta} /(r t)$, поэтому из определения величины 
$S$ следует, что

$$
\begin{aligned}
S & =\sum_{i=1}^{\lceil p / h\rceil-1} \sum_{j=1}^{c_{i}} \frac{h_{j}^{i} m_{j}^{i}}{(r t)^{N_{j}^{i}(1-\alpha)}} \leqslant \sum_{i=1}^{\lceil p / h\rceil-1} \sum_{j=1}^{c_{i}} \frac{h_{j}^{i} m_{j}^{i}(r t)^{1-\alpha}}{\left(h_{j}^{i}\right)^{(1-\beta)(1-\alpha)}} \\
& \leqslant \frac{r t}{(r t)^{\alpha}} \sum_{i=1}^{\lceil p / h\rceil-1} \sum_{j=1}^{c_{i}}\left(h_{j}^{i}\right)^{\beta+\alpha-\alpha \beta} m_{j}^{i} \leqslant \frac{r t}{z} h^{\beta+\alpha(1-\beta)} \sum_{i=1}^{\lceil p / h\rceil-1} p \\
& \leqslant \frac{z+\rho}{z} h^{\beta+\alpha(1-\beta)} \frac{p^{2}}{h} \leqslant(1+\rho) \frac{p^{2}}{h^{(1-\alpha)(1-\beta)}} \leqslant(1+\rho) \frac{p^{2}}{h^{\left(1-\log _{1+\rho} \rho\right)(1-\beta)}} .
\end{aligned}
$$

Из полученных оценок и (5.6) вытекает, что

$$
L_{A^{0}} \leqslant(1+\rho) \frac{p^{2}}{h^{\left(1-\log _{1+\rho} \rho\right)(1-\beta)}}+\frac{p^{2}}{h^{\beta}}+2^{h} h^{1-\beta} p+\frac{p h}{2}+\frac{h^{2}}{2} .
$$

Очевидно, что $\log _{2} p^{\varepsilon} \leqslant h \leqslant \log _{2} p^{\varepsilon}+1$, поэтому

$$
\begin{aligned}
L= & L_{A}+L_{A^{0}} \\
\leqslant & \frac{q}{\rho}+\frac{(1+\rho) p^{2}}{\left(\varepsilon \log _{2} p\right)^{\left(1-\log _{1+\rho} \rho\right)(1-\beta)}+\frac{p^{2}}{\left(\varepsilon \log _{2} p\right)^{\beta}}} \\
& \quad+\frac{2 p^{1+\varepsilon}\left(\left(\varepsilon \log _{2} p+1\right)\right.}{\left(\varepsilon \log _{2} p\right)}+\frac{p\left(\varepsilon \log _{2} p+1\right)}{2}+\frac{\left(\varepsilon \log _{2} p+1\right)^{2}}{2} \\
= & \frac{(1+\rho) p^{2}}{\rho}+\frac{p^{2}+2 p^{1+\varepsilon}}{\left(\varepsilon \log _{2} p\right)^{\left(1-\log _{1+\rho} \rho\right)(1-\beta)}+\frac{\left.p \log _{2} p\right)^{\beta}}{\left(\log _{2} p\right.}+\frac{p}{2}+\varepsilon O\left(\left(\log _{2} p\right)^{2}\right),} \\
& +2 p^{1+\varepsilon}\left(\varepsilon \log _{2} p\right)^{(1-\beta)}+\frac{p \varepsilon \log ^{2}}{2}
\end{aligned}
$$

то есть для сложности $L$ выполнено неравенство (5.4).

По теореме 1 построенному нами точному покрытию матрицы $M$ матрицами $A$ и $A^{0}$ соответствует точное покрытие графа $G$ графами $H$ и $H^{0}$ той же сложности. Следовательно, применив описанный алгоритм, можно построить точное покрытие графа $G$ графами $H$ и $H^{0}$ сложности $L$, для которой выполняется неравенство (5.4).

Теорема доказана.

Следствие 3. Если базис В с весом $\rho$ является слабо плотным, то

$$
l_{B}(q, p) \leqslant \frac{q}{\rho}+O\left(p^{2} /\left(\log _{2} p\right)^{\left.\beta_{0}\right)}\right)
$$

где

$$
\beta_{0}=\frac{1-\log _{\rho+1} \rho}{2+\log _{\rho+1} \rho}=\frac{\log _{1+\rho}((1+\rho / \rho))}{\log _{1+\rho}(1+\rho)^{2} \rho} .
$$

Доказательство. Пусть

$$
\beta_{0}=\frac{1-\log _{\rho+1} \rho}{2+\log _{\rho+1} \rho}
$$


тогда $0<\beta_{0}<1$. Выберем произвольное вещественное число $\varepsilon_{0}$ так, чтобы выполнялось неравенство $0<\varepsilon_{0}<\beta_{0}$, и положим в теореме $5 \varepsilon=\varepsilon_{0}, \beta=\beta_{0}$. Тогда

$$
\begin{aligned}
\left(1-\log _{1+\rho} \rho\right)(1-\beta) & =\log _{1+\rho} \frac{1+\rho}{\rho}\left(\frac{2+\log _{1+\rho} \rho}{2+\log _{1+\rho} \rho}-\frac{1-\log _{1+\rho} \rho}{2+\log _{1+\rho} \rho}\right) \\
& =\frac{\log _{1+\rho}((1+\rho) / \rho)}{\log _{1+\rho}(1+\rho)^{2}+\log _{1+\rho} \rho}=\frac{\log _{1+\rho}((1+\rho) / \rho)}{\log _{1+\rho}(1+\rho)^{2} \rho}=\beta,
\end{aligned}
$$

и из неравенства (5.4) вытекает, что для любого графа $G \in \mathscr{G}$ с $p$ вершинами и $q$ ребрами

$$
L_{\beta, \varepsilon}(q, p, \rho) \leqslant \frac{q}{\rho}+O\left(p^{2} /\left(\log _{2} p\right)^{\beta_{0}}\right) .
$$

Отсюда следует неравенство (5.7).

\section{Список литературы}

1. Емеличев В. А., Мельников О. И., Сарванов В. И., Тышкевич Р. И., Лекчии по теории графов. Наука, Москва, 1990.

2. Ложкина 3. С., О реберно-плотных базисах в классе графов. Вестник Нижегородского Госуниверситета, "Математическое моделирование и оптимальное управление" (2000) 22 №1.

3. Ложкина 3. С., О множестве почти плотных базисов в классе графов. Вестник Московского ун-та. Сер. 15, Выч. матем. и киберн. (2000) №3, 45-50.

4. Ложкина 3. С., О слабо плотных базисах в классе графов. Вестник Московского ун-та. Сер. 15 , Выч. матем. и киберн. (2001) №2, 47-53.

5. Ложкина 3. С., О сложности покрытия графов графами из спечиальных базисов. Диссертация на соискание ученой степени кандидата физ.-матем. наук, 2002.

Статья поступила 06.11.2003. 